\title{
Quantum theory of superfluorescence based on two-point correlation functions
}

\author{
Andrei Benediktovitch, ${ }^{1, *}$ Vinay P. Majety, ${ }^{2, \dagger}$ and Nina Rohringer ${ }^{1,3}$ \\ ${ }^{1}$ Center for Free-Electron Laser Science, DESY, Hamburg 22607, Germany \\ ${ }^{2}$ Max Planck Institute for the Structure and Dynamics of Matter, Hamburg 22607, Germany \\ ${ }^{3}$ Department of Physics, Universität Hamburg, Hamburg 22761, Germany
}

(Received 29 October 2018; published 22 January 2019)

\begin{abstract}
Irradiation of a medium by short intense pulses from x-ray (XUV) free-electron lasers can result in saturated photoionization of inner electronic shells. As a result an inversion of populations between core levels appears. The resulting fluorescent radiation can be amplified during its propagation through the inverted medium and results in intense, quasi-transform-limited radiation bursts. While the optical counterpart of this phenomena, known as superfluorescence, was intensively investigated, a generalized treatment is needed in the x-ray (XUV) domain, where the dynamics of pumping and evolution due to fast decay processes play a crucial role. To provide a general theoretical approach, we start from the fundamental, quantized minimal coupling Hamiltonian of light-matter interaction and after a series of approximations arrive at a closed system of equations for the two-point correlation function of atomic coherences and the two-time correlation function of the emitted field. The obtained formalism enables us to investigate collective spontaneous emission in various regimes. It is extended consistently to include incoherent processes that are relevant in the x-ray (XUV) domain. These processes are introduced into the formalism by corresponding Lindblad superoperators. The connection to other approaches is discussed and numerical examples related to recent experiments are presented.
\end{abstract}

DOI: 10.1103/PhysRevA.99.013839

\section{INTRODUCTION}

The interaction of $\mathrm{x}$-rays with matter has been providing unique information about the structure of matter at Angstrom length-scale for more than a century. One of the reasons for its success is the weak interaction between X-rays and atomic systems: The overwhelming majority of x-ray laboratory and synchrotron experiments can be described within linear response approaches. However, if a population inversion for transitions in the $\mathrm{x}$-ray range is created by some means, nonlinear stimulated emission processes will take place. Such a population inversion can, for example, be obtained by inner-shell photoionization, as was proposed in Ref. [1]. The power needed to sustain the population inversion scales unfavorably with the radiation frequency [2], hence the scheme was first demonstrated for transitions in the optical domainpumped by soft x-rays from a laser produced plasma [3]. There are a number of different schemes that were shown to produce population inversion for transitions in the XUV or soft X-ray domains, e.g., electron collision ionization in plasma [4], recombination of ions stripped by optical field (tunneling) ionization [5], and others; see Refs. [2,6,7] for further discussions. However, the stability and reproducibility of the population inversion was quite poor in many cases, and in addition, the amount of photons generated by stimulated radiation was quite low. The situation changed dramatically after the advent of x-ray free-electron lasers (XFEL). The inner-shell photoionization by XFEL pump and subsequent

\footnotetext{
*andrei.benediktovitch@desy.de

${ }^{\dagger}$ Present address: Indian Institute of Technology Tirupati, Tirupati, India.
}

stimulated emission was demonstrated first in gases [8] and then in solids [9] and solutions [10]. The emitted bursts of radiation were shown to have large photon numbers and a bandwidth much narrower than the pumping XFEL pulse. Hence, they are promising both for stimulated X-ray spectroscopy $[10,11]$ and for use as dedicated x-ray photon sources with unique characteristics that can be employed for further (pump-probe) experiments.

The process described above has been referred to as $\mathrm{x}$-ray lasing in many cases $[8,9,12]$. The shape of the gain region is determined by the overlap of the pumping $\mathrm{x}$-ray beam and the driven medium. Focusing the x-rays into a target results in an elongated, cylindrical shape. Comparing the process to traditional lasing in the optical regime, there are a number of marked differences: (i) the $\mathrm{x}$-ray pumping is applied in form of a short pulse propagating through the medium, (ii) there is no feedback system employed, (iii) there can be numerous loss and decay processes that act on timescales comparable to the emission process, resulting in transient gain. Each emission starts from noiselike spontaneous radiation. The portion of radiation emitted along the axis of the beam is amplified during a single-pass propagation through the inverted medium and, if the number of involved inverted atoms is large enough, the stored energy is radiated within a short, intense pulse. A comparable phenomenon in the optical domain is referred to as superfluorescence, and has seen extensive experimental investigation; see Refs. [13-16] for early examples. Notably, the term superfluorescence is often used synonymously with superradiance. Furthermore, the crossover from superfluorescence to amplified spontaneous emission is not sharp [16]. Hence, theory becomes essential for the interpretation of experimental results. 
Theoretical modeling of the phenomena described above, which we will generally refer to as superfluorescence, is an involved problem and has been studied extensively (cf. Refs. [17-20] and references therein). The complexity of the problem was described in Ref. [18], p. 336, as follows: "The problem of superradiance in free space is in general a problem of three-dimensional nonlinear diffraction theory, further complicated by the quantum nature of the radiated field at the early stage of the emission process." Hence, theoretical models used to describe experimental data typically have to rely on a number of approximations to reduce the complexity of the problem. The range of validity is conditioned by the parameters of the system under study. In many cases, a onedimensional semiclassical Maxwell-Bloch model is used for the description of x-ray (XUV) experiments based on XFEL pumping [10-12,21,22] or plasma-based pumping [23-27]. The reduction to a one-dimensional problem is justified by the shape of the excited medium, which resembles an extensively elongated cylinder. The nonlinear nature of the stimulated process can also be captured in the Maxwell-Bloch formalism. However, while having a clear interpretation and being comparatively easy to simulate numerically, the semiclassical 1D Maxwell-Bloch equations are not able to describe the initial stage of the amplification process. Namely, the semiclassical 1D Maxwell-Bloch equations form a homogeneous system of differential equations. Thus if all atoms are initially in the inverted state and neither field nor polarization is present at that moment, there will also not be any field or polarization at later times. Here, accounting for the quantum nature of the radiated field is an unavoidable ingredient as only this will allow the system to relax by means of spontaneous emission.

Several approaches have been proposed to extend the semiclassical Maxwell-Bloch formalism to account for spontaneous emission. One way is to assume a fixed, small value of the polarization at the initial time. In terms of a Bloch sphere representation, this corresponds to the introduction of a small tipping angle of the Bloch vector from North pole. In the 1970 s, multiple ways to calculate the actual value of this tipping angle were proposed [28-32]; in Ref. [15], these approaches were compared and an experimental confirmation for the values obtained in Refs. [29,32] was given. However, even though such a tipping angle approach can be sufficient to reproduce the general shape of the emitted pulses, some important features of the superfluorescent emission are missing in this approach. In particular, it fails to account for the strong fluctuations in shape and arrival times that superfluorescent pulses exhibit as a consequence of their origin in quantum fluctuations. These can be described more adequately in a framework such as proposed in Ref. [33]. There, the observables are obtained from an ensemble of solutions to the Maxwell-Bloch equations corresponding to random initial polarizations. The distribution of the initial polarization is obtained from properties of the polarization operator assuming all atoms to be in the excited state. In this approach, the process that excites the atoms is explicitly separated from the superfluorescence dynamics. However, for Xray (XUV) experiments the timescales for processes initiated by pumping and the timescale for the superfluorescence dynamics can in general be of the same order. The simultaneous description of evolution due to pumping and superfluorescent emission can be achieved, if random noise terms are considered in the equations for polarization or polarization and field instead of random initial conditions. These noise terms have been derived from first principles using phase space approaches in Refs. [34,35] and give contributions to both the polarization and the emitted field. The noise has a multiplicative structure and-in general-can lead to unstable numerical behavior of simulations after finite propagation times. In Ref. [23], the noise terms for the polarization were derived semiphenomenologically. Here, we avoid the phenomenological introduction of terms into the MaxwellBloch equations and instead start from a quantum-mechanical Hamiltonian. Following a series of approximations, we arrive at equations of motion for the correlation function of atomic coherences and the field correlation function. In this formulation, the spontaneous and stimulated emission enter on the same footing, hence the crossover between them can be analyzed straightforwardly.

The paper is organized as follows: in Sec. II, we start from the basic Hamiltonian that describes the interaction of two-level atoms with the electromagnetic field arriving at a system of integrodifferential equations for the level populations, the correlation function of atomic coherences, and the correlation function of the field. In Sec. III, these equations are generalized to include a number of incoherent processes, which are introduced by means of Lindblad superoperators. In Sec. IV, we discuss the crossover to spontaneous emission and to the semiclassical Maxwell-Bloch equations. Furthermore, we compare our approach to the Maxwell-Bloch equations with stochastic noise terms and present a modeling example of a realistic system. Details of the calculation that were skipped in the main text are presented in Appendices A-F. SI units are used throughout this article.

\section{DERIVATION OF EVOLUTION EQUATIONS OF THE CORRELATION FUNCTION}

To study the fundamental features of collective spontaneous emission, we employ the following model. Consider an elongated, roughly cylindrical medium consisting of a large number of two-level atoms. We assume an ideal sweptgain pumping of the system, that is a population inversion is created quasi-instantaneously by a pump-pulse front that transverses the medium with speed of light [36-38]. The temporal evolution of the atomic ensemble in interaction with (vacuum) electromagnetic field modes is determined by the well-known Hamiltonian [39]:

$$
\begin{aligned}
\hat{H}= & \sum_{a} \hbar \Omega \hat{\sigma}_{z}^{(a)}+\int d^{3} \vec{k} \sum_{s} \hbar \omega_{\vec{k}} \hat{a}_{\vec{k}, s}^{\dagger} \hat{a}_{\vec{k}, s} \\
& +\int d^{3} \vec{k} \sum_{a, s} \hbar\left[g_{\vec{k}, s} e^{i \vec{k} \vec{r}_{a}} \hat{\sigma}_{+}^{(a)} \hat{a}_{\vec{k}, s}+g_{\vec{k}, s}^{*} e^{-i \vec{k} \vec{r}_{a}} \hat{a}_{\vec{k}, s}^{\dagger} \hat{\sigma}_{-}^{(a)}\right] .
\end{aligned}
$$

Here, $\Omega$ denotes the frequency of the transition between the ground $|g\rangle$ and excited $|e\rangle$ state of each atom. The operators $\hat{\sigma}_{z,+,-}^{(a)}$ correspond to observables of two-level atom $a: \hat{\sigma}_{z}$ corresponds to the population inversion,

$$
\hat{\sigma}_{z}=\frac{1}{2}(|e\rangle\langle e|-| g\rangle\langle g|),
$$


and $\hat{\sigma}_{+,-}$corresponds to atomic coherences,

$$
\hat{\sigma}_{+}=|e\rangle\left\langle g\left|, \hat{\sigma}_{-}=\right| g\right\rangle\langle e| .
$$

The operators $\hat{a}_{\vec{k}, s}^{\dagger}, \hat{a}_{\vec{k}, s}$ are creation and annihilation operators for photons in the electromagnetic field mode with wave vector $\vec{k}$, frequency $\omega_{\vec{k}}$, and polarization $s ; \vec{r}_{a}$ gives the position of atom $a$ and $g_{\vec{k}, s}$ is the coupling constant for an atom and the electromagnetic field due to the $\vec{A} \cdot \vec{p}$ interaction term. It is given as

$$
g_{\vec{k}, s}=\frac{e}{m} \sqrt{\frac{1}{2(2 \pi)^{3} \epsilon_{0} \hbar \omega_{\vec{k}}}} \vec{p} \cdot \vec{e}_{\vec{k}, s}, \quad \text { with } \quad \vec{p}=\langle e|\hat{\vec{p}}| g\rangle,
$$

where $\vec{e}_{\vec{k}, s}$ is a unit polarization vector. Notably, the interaction Hamiltonian in Eq. (1) implies the rotating wave approximation. Furthermore, the dipole approximation was invoked in Eq. (4), which is valid for core-shell excitations in the x-ray domain; see Ref. [40].

\section{A. Heisenberg-Langevin equations}

Our aim is to obtain a closed system of equations for the atomic and field observables. To accomplish this, we need the generic equation of motion for these quantities first. Following the usual derivation of Heisenberg-Langevin equations [39,41], we arrive at (see the detailed derivation in Appendix A)

$$
\begin{aligned}
\frac{d \hat{\sigma}_{-}^{(a)}(t)}{d t}= & -i \Omega \hat{\sigma}_{-}^{(a)}(t)-\frac{\Gamma_{\mathrm{sp}}}{2} \hat{\sigma}_{-}^{(a)}(t) \\
& +\frac{2 i e}{m \hbar c} \hat{\sigma}_{z}^{(a)}(t) \vec{p} \cdot \overrightarrow{\hat{A}}_{+}^{(a)}(t)+\hat{F}_{-}^{(a)}(t) \\
\frac{d \hat{\sigma}_{z}^{(a)}(t)}{d t}=- & \Gamma_{\mathrm{sp}} \hat{\sigma}_{+}^{(a)}(t) \hat{\sigma}_{-}^{(a)}(t)+\frac{i e}{m \hbar c}\left[\vec{p}^{*} \cdot \overrightarrow{\hat{A}}_{-}^{(a)}(t) \hat{\sigma}_{-}^{(a)}(t)\right. \\
- & \left.\hat{\sigma}_{+}^{(a)}(t) \vec{p} \cdot \overrightarrow{\hat{A}}_{+}^{(a)}(t)\right]+\hat{F}_{z}^{(a)}(t) \\
\overrightarrow{\hat{A}}_{+}^{(a)}(t)= & -\frac{i e c}{16 \pi^{3} m \epsilon_{0}} \int d^{3} \vec{k} \sum_{s} \frac{1}{\omega_{\vec{k}}} \vec{e}_{\vec{k}, s}\left(\vec{e}_{\vec{k}, s}^{*} \cdot \vec{p}^{*}\right) \\
& \times \int_{0}^{t} d t^{\prime} \sum_{b \neq a} e^{i\left(\vec{k}\left(\overrightarrow{r_{a}}-\vec{r}_{b}\right)-\omega_{\vec{k}}\left(t-t^{\prime}\right)\right)} \hat{\sigma}_{-}^{(b)}\left(t^{\prime}\right)
\end{aligned}
$$

Here, $\overrightarrow{\hat{A}}_{+}^{(a)}$ is the positive-frequency part of the vector potential at the position of atom $a$ due to all other atoms, $\hat{F}_{z, \pm}^{(a)}(t)$ are stochastic Langevin terms due to interaction with vacuum field (see the details in Appendix A), $\Gamma_{\mathrm{sp}}=\frac{e^{2} \Omega|\vec{p}|^{2}}{3 \pi \epsilon_{0} \hbar m^{2} c^{3}}$ is the spontaneous emission rate.

Up to this point, no approximations were made regarding the emitted electromagnetic field. Due to this generality, Eqs. (5)-(7) are non-Markovian with respect to interaction among the atoms: the evolution of a given atom $a$ is conditioned by operator values of all other atoms at all previous points in time. These operator equations, however, are still of limited use for treating a large number of atoms. To proceed, let us make the following approximations: i) omit the polarization properties of the radiation and ii) restrict the description of the field to one dimension. The first approximation is equivalent to the assumption that all atoms have the vector of their dipole transition matrix element oriented along the same (fixed) direction. This direction is assumed to be orthogonal to the propagation direction of the pump field and is further considered as the polarization direction of the induced field. This assumption is not crucial for the developed formalism, but it essentially simplifies the equations. To perform the reduction to a $1 \mathrm{D}$ model let us take into account that only those modes of the field that have their wave vectors roughly aligned with the axis of the cylindrical medium will interact with large number of atoms and thus will be amplified. Based on this consideration, let us assume that all waves with a wave vector oriented along the system $z$ axis within a small solid angle $\Delta o$ have the same magnitude. Waves with a wave vector outside $\Delta o$ are neglected. In particular, waves are neglected that would be counterpropagating to the swept pumping field. These assumptions correspond to the following way of calculating the integral over wave vectors in Eq. (7):

$$
\int d^{3} \vec{k} \rightarrow \Delta o \int_{0}^{\infty} d \omega \frac{\omega^{2}}{c^{3}} .
$$

The actual value of $\Delta o$ to be used in calculations can be estimated from geometrical considerations as $\Delta o_{g} \sim \pi R^{2} / L^{2}$ where $R$ is radius of the medium and $L$ is its length. However, if $\Delta o_{g}$ exceeds the solid angle $\Delta o_{d} \sim \lambda^{2} / R^{2}$, diffraction effects become important and one can expect that the emission decomposes into independently radiating regions of size $\Delta o_{d}$, see Ref. [18] for further discussion.

With help of (8), performing similar steps as in the derivation of (A8), and omitting the vectorial properties of the field one obtains

$$
\begin{aligned}
\hat{A}_{+}^{(a)}(t)= & -\frac{i \Delta o e p^{*} \Omega}{8 \pi^{2} \epsilon_{0} m c^{2}} \int_{0}^{t} d t^{\prime} \sum_{b \neq a} \delta\left[t-\frac{z_{a}}{c}\right. \\
& \left.-\left(t^{\prime}-\frac{z_{b}}{c}\right)\right] \hat{\sigma}_{-}^{(b)}\left(t^{\prime}\right) .
\end{aligned}
$$

Due to the assumption of swept-gain pumping, which propagates with speed $c$ through the medium, the non-trivial evolution of atom $a$ starts at the time $z_{a} / c$. Hence, all atomic operators $\hat{\sigma}^{(a)}$ have a time dependence of the form $\hat{\sigma}^{(a)}(t-$ $\left.\frac{z a}{c}\right)$. Let us change variables to the retarded time $\tau$, defined for each atom $a$ as $\tau=t-\frac{z_{a}}{c}$. In terms of this variable one obtains from Eq. (9)

$$
\hat{A}_{+}^{(a)}(\tau)=-\frac{i \Delta o e p \Omega}{16 \pi^{2} \epsilon_{0} m c^{2}} \sum_{b<a} \hat{\sigma}_{-}^{(b)}(\tau)
$$

Here, $\sum_{b<a}$ means that one has to sum over atoms $b$ positioned before (with respect to pump propagation) the considered atom $a$. With the help of Eq. (10), Eqs. (5) and (6) take the form

$$
\begin{aligned}
\frac{d \hat{\sigma}_{-}^{(a)}(\tau)}{d \tau}= & -i \Omega \hat{\sigma}_{-}^{(a)}(\tau)-\frac{\Gamma_{\mathrm{sp}}}{2} \hat{\sigma}_{-}^{(a)}(\tau) \\
& +\frac{3 \Delta o}{8 \pi} \Gamma_{\mathrm{sp}} \sum_{b<a} \hat{\sigma}_{z}^{(a)}(\tau) \hat{\sigma}_{-}^{(b)}(\tau)+\hat{F}_{-}^{(a)}(\tau),
\end{aligned}
$$




$$
\begin{aligned}
\frac{d \hat{\sigma}_{z}^{(a)}(\tau)}{d \tau}= & -\Gamma_{\mathrm{sp}} \hat{\sigma}_{+}^{(a)}(\tau) \hat{\sigma}_{-}^{(a)}(\tau) \\
& -\frac{3 \Delta o}{16 \pi} \Gamma_{\mathrm{sp}} \sum_{b<a}\left[\hat{\sigma}_{+}^{(a)}(\tau) \hat{\sigma}_{-}^{(b)}(\tau)+\hat{\sigma}_{+}^{(b)}(\tau) \hat{\sigma}_{-}^{(a)}(\tau)\right] \\
& +\hat{F}_{z}^{(a)}(\tau)
\end{aligned}
$$

\section{B. Equations for atomic observables}

The operator Eqs. (11) and (12) can be used to obtain equations for their mean values. In the following we will obtain equations for the expectation values of the atomic coherences, population inversion and we will also introduce a correlation function of atomic coherences.

\section{Coherences}

The mean value of atomic coherences is given by $\left\langle\hat{\sigma}_{-}^{(a)}\right\rangle=$ $\operatorname{Tr}\left(\hat{\sigma}_{-}^{(a)} \hat{\rho}^{(a)}\right)=\rho_{e g}^{(a)},\left\langle\hat{\sigma}_{+}^{(a)}\right\rangle=\rho_{e g}^{(a) *}$, where $\hat{\rho}^{(a)}$ denotes the one-atom density matrix for atom $a$. Assuming factorization of the operators acting on different atoms (this is valid at the initial moment of time) and taking the mean value of Eq. (11), we end up with a homogeneous equation for each $\left\langle\hat{\sigma}_{-}^{(a)}\right\rangle$. If initially there is no coherent excitation in the system $\left\langle\hat{\sigma}_{-}^{(a)}(\tau=\right.$ $0)\rangle=0$, it will remain the same during the evolution:

$$
\left\langle\hat{\sigma}_{ \pm}^{(a)}(\tau)\right\rangle=0 .
$$

\section{Population inversion}

We define the population inversion to be $\left\langle\hat{\sigma}_{z}^{(a)}\right\rangle=\frac{1}{2}\left(\rho_{e e}^{(a)}-\right.$ $\left.\rho_{g g}^{(a)}\right)$, where $\rho_{e e}^{(a)}$ and $\rho_{g g}^{(a)}$ are the populations of the ground and excited states of atom $a$. From Eq. (12) one obtains

$$
\begin{aligned}
\frac{d\left\langle\hat{\sigma}_{z}^{(a)}(\tau)\right\rangle}{d \tau}= & -\Gamma_{\mathrm{sp}} \rho_{e e}^{(a)}(\tau)-\frac{3 \Delta o}{16 \pi} \Gamma_{\mathrm{sp}} \sum_{b<a}\left[\left\langle\hat{\sigma}_{+}^{(a)}(\tau) \hat{\sigma}_{-}^{(b)}(\tau)\right\rangle\right. \\
& \left.+\left\langle\hat{\sigma}_{+}^{(b)}(\tau) \hat{\sigma}_{-}^{(a)}(\tau)\right\rangle\right] .
\end{aligned}
$$

Here, we have taken into account the property $\left\langle\hat{\sigma}_{+}^{(a)}(\tau) \hat{\sigma}_{-}^{(a)}(\tau)\right\rangle=\rho_{e e}^{(a)}(\tau)$ of the coherence operators, which follows immediately from Eq. (3). The noise term $\hat{F}_{z}^{(a)}(\tau)$ has disappeared due to the property Eq. (A11). Hence, Eq. (14) is a nonstochastic $c$-number differential equation, as well as other equations for observables.

Notably, Eq. (14) involves the quantity $\left\langle\hat{\sigma}_{+}^{(a)}(\tau) \hat{\sigma}_{-}^{(b)}(\tau)\right\rangle$, which we shall subsequently consider in more detail.

\section{Correlation function of atomic coherences}

The value of $\left\langle\hat{\sigma}_{+}^{(a)}(\tau) \hat{\sigma}_{-}^{(b)}(\tau)\right\rangle$ quantifies the joint probability for atoms $a$ and $b$ to exhibit coherence. It is related to the two-atom density matrix $\hat{\rho}^{(a b)}$ as

$$
\left.\left\langle\hat{\sigma}_{+}^{(a)}(\tau) \hat{\sigma}_{-}^{(b)}(\tau)\right\rangle=\operatorname{Tr}\left[\hat{\rho}^{(a b)}(\tau) \hat{\sigma}_{+}^{(a)}(\tau) \hat{\sigma}_{-}^{(b)}(\tau)\right\rangle\right]=\rho_{g_{a} e_{b}, e_{a} g_{b}}^{(a b)} .
$$

Similarly to the quantity $\left\langle\hat{E}_{-}\left(\vec{r}_{1}, t_{1}\right) \hat{E}_{+}\left(\vec{r}_{2}, t_{2}\right)\right\rangle=$ $\operatorname{Tr}\left[\hat{\rho}^{(F)} \hat{E}_{-}\left(\vec{r}_{1}, t_{1}\right) \hat{E}_{+}\left(\vec{r}_{2}, t_{2}\right)\right]$-being named correlation function of the field $[39,42]$ we can refer to Eq. (15) as the correlation function of atomic coherences. Since the two-level atom polarization is proportional to the atomic coherence, the quantity Eq. (15) gives the correlation of atomic polarizations up to a factor $|p|^{2}$.

The equation of motion of Eq. (15) can be obtained based on Eq. (11), see the details in Appendix B:

$$
\begin{aligned}
\frac{d\left\langle\hat{\sigma}_{+}^{(a)}(\tau) \hat{\sigma}_{-}^{(b)}(\tau)\right\rangle}{d \tau}= & -\Gamma_{\mathrm{sp}}\left\langle\hat{\sigma}_{+}^{(a)}(\tau) \hat{\sigma}_{-}^{(b)}(\tau)\right\rangle \\
& +\frac{3 \Delta o}{8 \pi} \Gamma_{\mathrm{sp}}\left[\sum_{c<a}\left\langle\hat{\sigma}_{+}^{(c)}(\tau) \hat{\sigma}_{z}^{(a)}(\tau) \hat{\sigma}_{-}^{(b)}(\tau)\right\rangle\right. \\
& \left.+\sum_{c<b}\left\langle\hat{\sigma}_{+}^{(a)}(\tau) \hat{\sigma}_{z}^{(b)}(\tau) \hat{\sigma}_{-}^{(c)}(\tau)\right\rangle\right] .
\end{aligned}
$$

Equations (14) and (16) do not yet form closed system of equations, because of the triple operator products on the right-hand side of Eq. (16). It should be noted that similar equations for triple operator products would couple to quartic operator product and so on. The hierarchy of these equations would reflect the BBGKY hierarchy of reduced density matrix equations [43]. To obtain a finite and closed system of equations, we factorize triple operator products as

$$
\left\langle\hat{\sigma}_{+}^{(a)}(\tau) \hat{\sigma}_{z}^{(b)}(\tau) \hat{\sigma}_{-}^{(c)}(\tau)\right\rangle \underset{b \neq a, c}{\approx}\left\langle\hat{\sigma}_{z}^{(b)}(\tau)\right\rangle\left\langle\hat{\sigma}_{+}^{(a)}(\tau) \hat{\sigma}_{-}^{(c)}(\tau)\right\rangle .
$$

The triple product factorization generally enables the description of a large class of phenomena and form the basis for most important kinetic equations [43]. With respect to superfluorescence type of problems, it was demonstrated numerically that dropping third-order cumulants (equivalent to the factorization (17)) does not affect the solution of the steady-state superradiance problem $[44,45]$. Performing the factorization (17) and treating the terms with $c=b$ or $c=a$ on the right-hand side of Eq. (16) separately, we obtain

$$
\begin{aligned}
\frac{d\left\langle\hat{\sigma}_{+}^{(a)}(\tau) \hat{\sigma}_{-}^{(b)}(\tau)\right\rangle}{d \tau}= & -\Gamma_{\mathrm{sp}}\left\langle\hat{\sigma}_{+}^{(a)}(\tau) \hat{\sigma}_{-}^{(b)}(\tau)\right\rangle+\frac{3 \Delta o}{8 \pi} \Gamma_{\mathrm{sp}}\left[\sum_{c<a, c \neq b}\left\langle\hat{\sigma}_{z}^{(a)}(\tau)\right\rangle\left\langle\hat{\sigma}_{+}^{(c)}(\tau) \hat{\sigma}_{-}^{(b)}(\tau)\right\rangle+\sum_{c<b, c \neq a}\left\langle\hat{\sigma}_{z}^{(b)}(\tau)\right\rangle\left\langle\hat{\sigma}_{+}^{(a)}(\tau) \hat{\sigma}_{-}^{(c)}(\tau)\right\rangle\right] \\
& +\frac{3 \Delta o}{8 \pi} \Gamma_{\mathrm{sp}}\left[\left\langle\hat{\sigma}_{z}^{(a)}(\tau)\right) \rho_{e e}^{(b)}(\tau) \Theta\left(z_{a}-z_{b}\right)+\left\langle\hat{\sigma}_{z}^{(b)}(\tau)\right\rangle \rho_{e e}^{(a)}(\tau) \Theta\left(z_{b}-z_{a}\right)\right],
\end{aligned}
$$

where $\Theta(z)$ is the Heaviside function. Equations (14) and (18) form a closed set of equations for the evolution of the electronic degrees of freedom.

\section{Equations for field observables}

In most of the experiments dealing with x-ray (XUV) collective emission, the measured observables are related to 
the properties of the emitted electromagnetic field. Hence, we consider in more detail properties of the emitted field.

\section{Slowly varying amplitude of the vector potential}

The vector potential is given by Eq. (10); due to Eq. (13) one straightforwardly obtains

$$
\left\langle\hat{A}_{ \pm}^{(a)}\right\rangle=0
$$

This implies that the ensemble average emitted field cannot be described by a classical amplitude. Instead, the description should be given in terms of statistical properties of the field.

\section{Intensity}

The statistical property of primary interest is the intensity of the emitted field. Let us quantify this by the number of photons emitted per solid angle $\Delta o$ per cross section of the system per unit time. Thus, for the intensity at the position of atom $a$, we can take the value of the Poynting vector and normalize by $\Delta o \hbar \omega$. Applying normal ordering, we obtain

$$
\begin{aligned}
I^{(a)}(\tau) & =\frac{2 \epsilon_{0} \omega}{\Delta o \hbar c}\left\langle\hat{A}_{-}^{(a)}(\tau) \hat{A}_{+}^{(a)}(\tau)\right\rangle \\
& =\frac{3 \Delta o}{32 \pi \lambda^{2}} \Gamma_{\mathrm{sp}} \sum_{b<a, c<a}\left\langle\hat{\sigma}_{+}^{(b)}(\tau) \hat{\sigma}_{-}^{(c)}(\tau)\right\rangle .
\end{aligned}
$$

Here, we have used Eq. (10) to obtain the second equation, employing also the spontaneous emission rate $\Gamma_{\text {sp }}$ Eq. (A8) and the radiation wavelength $\lambda$.

Hence, we can directly evaluate the intensity as soon as the correlations between atomic coherences are known.

\section{Field correlation function and spectral intensity}

Another important field characteristic is its spectral intensity. According to the Wiener - Khinchin theorem, this quantity can be obtained from the Fourier transformation of the field correlation function. Using the same normalization as for the intensity Eq. (20) and expressing the field correlation function in terms of atomic variables, we find

$$
\begin{aligned}
G^{(a)}\left(\tau_{1}, \tau_{2}\right)= & \frac{2 \epsilon_{0} \omega}{\Delta o \hbar c}\left\langle\hat{A}_{-}^{(a)}\left(\tau_{1}\right) \hat{A}_{+}^{(a)}\left(\tau_{2}\right)\right\rangle \\
& =\frac{3 \Delta o}{32 \pi \lambda^{2}} \Gamma_{\mathrm{sp}} \sum_{b<a, c<a}\left\langle\hat{\sigma}_{+}^{(b)}\left(\tau_{1}\right) \hat{\sigma}_{-}^{(c)}\left(\tau_{2}\right)\right\rangle .
\end{aligned}
$$

The two-time correlation function of atomic coherences $\left\langle\hat{\sigma}_{+}^{(b)}\left(\tau_{1}\right) \hat{\sigma}_{-}^{(c)}\left(\tau_{2}\right)\right\rangle$ cannot be related straightforwardly to the one-time correlation of atomic coherences Eq. (15) as obtained from the solution of Eq. (18). The quantum regression theorem, that is typically employed for establishing the connection between one-time and two-time correlations, requires the solution for one-atom averages [39]. In our case, the equations for one-atom averages that follow from Eq. (11) are nonlinear and the analytical solution is not straightforward.

In an alternative approach, we reformulate the problem of obtaining the field correlation function Eq. (21) into the problem of propagating it in space. For the difference between the field correlation function at position $z_{a}$ of atom $a$ and the position $z_{a+1}$ of neighboring atom $a+1$, we obtain the following expression within the approximation Eq. (17) (see details in Appendix C):

$$
\begin{aligned}
G^{(a+1)}\left(\tau_{1}, \tau_{2}\right)-G^{(a)}\left(\tau_{1}, \tau_{2}\right) \\
=\frac{3 \Delta o}{8 \pi} \Gamma_{\mathrm{sp}}\left(\int_{0}^{\tau_{1}} d \tau_{1}^{\prime} e^{-\left(-i \Omega+\frac{\Gamma_{\mathrm{sp}}}{2}\right)\left(\tau_{1}-\tau_{1}^{\prime}\right)}\left\langle\hat{\sigma}_{z}^{(a)}\left(\tau_{1}^{\prime}\right)\right\rangle G^{(a)}\left(\tau_{1}^{\prime}, \tau_{2}\right)\right. \\
\left.\quad+\int_{0}^{\tau_{2}} d \tau_{2}^{\prime} e^{-\left(i \Omega+\frac{\Gamma_{\mathrm{sp}}}{2}\right)\left(\tau_{2}-\tau_{2}^{\prime}\right)}\left\langle\hat{\sigma}_{z}^{(a)}\left(\tau_{2}^{\prime}\right)\right\rangle G^{(a)}\left(\tau_{1}, \tau_{2}^{\prime}\right)\right) \\
\quad+\frac{3 \Delta o}{32 \pi \lambda^{2}} \Gamma_{\mathrm{sp}} \rho_{e e}^{(a)}(0) e^{i \Omega\left(\tau_{1}-\tau_{2}\right)} e^{-\frac{\Gamma_{\mathrm{sp}}}{2}\left(\tau_{1}+\tau_{2}\right)} .
\end{aligned}
$$

\section{Representation in continuous variables}

The set of Eqs. (14), (18), and (22) forms a closed system of equations that enables us to derive the intensity and spectral properties of the emitted superfluorescence field. Since we are interested in macroscopic systems involving large numbers of atoms, it is advantageous to represent the obtained equations in terms of continuous quantities. With this aim, we introduce

$$
\begin{aligned}
& \rho_{\mathrm{inv}}(z, \tau):=\frac{1}{n \Delta z} \sum_{\substack{a: z<z_{a}<z+\Delta z \\
S\left(z_{1}, z_{2}, \tau\right)}} 2\left\langle\hat{\sigma}_{z}^{(a)}(\tau)\right\rangle \\
& G\left(z, \tau_{1}, \tau_{2}\right):=\frac{1}{(n \Delta z)^{2}} \sum_{\substack{a: z_{1}<z_{a}<z_{1}+\Delta z \\
b: z_{2}<z_{b}<z_{2}+\Delta z}}\left\langle\hat{\sigma}_{+}^{(a)}(\tau) \hat{\sigma}_{-}^{(b)}(\tau)\right\rangle \\
& \sum_{a: z<z_{a}<z+\Delta z} G^{(a)}\left(\tau_{1}, \tau_{2}\right)
\end{aligned}
$$

Here, $n=n_{v} \pi R^{2}$ is the linear concentration of atoms $\left(n_{v}\right.$ is volume concentration, $R$ is radius of cylindrical excited medium), $\rho_{\text {inv }}$ is the population inversion, $S$ is the correlation function of atomic coherences, and $G$ is the correlation function of the field. As before, knowledge of the correlation function of atomic coherences is only required explicitly for different atoms $a \neq b$. In terms of these new variables, the system of Eqs. (14), (18), and (22) can be represented as the following system of integrodifferential equations:

$$
\begin{gathered}
\frac{\partial \rho_{\mathrm{inv}}(z, \tau)}{\partial \tau}=-2 \Gamma_{\mathrm{sp}} \rho_{e e}(z, \tau)-\frac{3 \Delta o}{4 \pi} \Gamma_{\mathrm{sp}} n \int_{0}^{z} d z^{\prime} S\left(z, z^{\prime}, \tau\right), \\
\frac{\partial S\left(z_{1}, z_{2}, \tau\right)}{\partial \tau}=-\Gamma_{\mathrm{sp}} S\left(z_{1}, z_{2}, \tau\right)+\frac{3 \Delta o}{16 \pi} \Gamma_{\mathrm{sp}} n_{1}\left[\rho_{\text {inv }}\left(z_{1}, \tau\right) \int_{0}^{z_{1}} d z_{1}^{\prime} S\left(z_{1}^{\prime}, z_{2}, \tau\right)+\rho_{\mathrm{inv}}\left(z_{2}, \tau\right) \int_{0}^{z_{2}} d z_{2}^{\prime} S\left(z_{1}, z_{2}^{\prime}, \tau\right)\right] \\
+\frac{3 \Delta o}{16 \pi} \Gamma_{\mathrm{sp}}\left[\rho_{\mathrm{inv}}\left(z_{1}, \tau\right) \rho_{e e}\left(z_{2}, \tau\right) \Theta\left(z_{1}-z_{2}\right)+\rho_{\mathrm{inv}}\left(z_{2}, \tau\right) \rho_{e e}\left(z_{1}, \tau\right) \Theta\left(z_{2}-z_{1}\right)\right],
\end{gathered}
$$




$$
\begin{aligned}
\frac{\partial G\left(z, \tau_{1}, \tau_{2}\right)}{\partial z}= & \frac{3 \Delta o}{16 \pi} \Gamma_{\mathrm{sp}} n\left[\int_{0}^{\tau_{1}} d \tau_{1}^{\prime} e^{\left(i \Omega-\frac{\Gamma_{\mathrm{sp}}}{2}\right)\left(\tau_{1}-\tau_{1}^{\prime}\right)} \rho_{\mathrm{inv}}\left(z, \tau_{1}^{\prime}\right) G\left(z, \tau_{1}^{\prime}, \tau_{2}\right)+\int_{0}^{\tau_{2}} d \tau_{2}^{\prime} e^{-\left(i \Omega+\frac{\Gamma_{\mathrm{sp}}}{2}\right)\left(\tau_{2}-\tau_{2}^{\prime}\right)} \rho_{\mathrm{inv}}\left(z, \tau_{2}^{\prime}\right) G\left(z, \tau_{1}, \tau_{2}^{\prime}\right)\right] \\
& +\frac{3 \Delta o}{32 \pi \lambda^{2}} \Gamma_{\mathrm{sp}} n \rho_{e e}(z, 0) e^{i \Omega\left(\tau_{1}-\tau_{2}\right)} e^{-\frac{\Gamma_{\mathrm{sp}}}{2}\left(\tau_{1}+\tau_{2}\right)} .
\end{aligned}
$$

Here, the occupation of excited states $\rho_{e e}(z, \tau)$ can be related to the population inversion as $\rho_{e e}(z, \tau)=\left[1+\rho_{\text {inv }}(z, \tau)\right] / 2$ for a pure two-level system. Equations similar to Eqs. (24) and (25) were obtained in Ref. [46] in the frame of the unrestricted Markovian master equation. Such an approach, however, is only capable to provide single-time observables. The two-time field correlation function, which is needed to investigate spectral properties, requires special treatment as in our case by Eq. (26). Provided one is interested only in intensity profiles, then instead of solving Eq. (26) one can also express the intensity directly in terms of solutions of Eqs. (24) and (25). Namely, one can use Eq. (20), separate terms with $b=c$ and express the result in terms of Eq. (23):

$$
\begin{aligned}
I(z, \tau)= & \frac{3 \Delta o}{32 \pi \lambda^{2}} \Gamma_{\mathrm{sp}}\left[n^{2} \int_{0}^{z} d z_{1}^{\prime} \int_{0}^{z} d z_{2}^{\prime} S\left(z_{1}^{\prime}, z_{2}^{\prime}, \tau\right)\right. \\
& \left.+n \int_{0}^{z} d z^{\prime} \rho_{e e}\left(z^{\prime}, \tau\right)\right] .
\end{aligned}
$$

\section{INCLUSION OF PUMP PROCESS AND INCOHERENT PROCESSES}

\section{A. Incoherent processes relevant in x-ray (XUV) domain}

The system of Eqs. (24)-(26) is capable of describing collective spontaneous emission from a medium that consists of pure two-level atoms. It also accounts for the swept instantaneous pumping of the atoms into their excited states. It is also possible to treat more general cases, where the excited state occupation resulting from the pump is position dependent through the use of any function $\rho_{e e}(z, 0)$ as the initial condition. So far, however, we have always assumed that the condition $\rho_{e e}(z, t)+\rho_{g g}(z, t)=1$ is satisfied, which is insufficient for describing most of the experiments on collective spontaneous emission in x-ray (XUV) domain. There, the levels, between which the population inversion occurs, are populated and/or depleted as a result of multiple incoherent processes. Most significantly, this includes photoionization and can include Auger decay. Depending on the actual parameters of the system, the timescales of these processes can be comparable to the timescale of collective spontaneous emission. A strong point of our approach is that it can be generalized in a regular way to include processes of incoherent pumping, depletion, and coherence decay, all of which affect the dynamics of the considered transition.

To provide a formalism that can be coupled to further rate equations describing the evolution of atoms in the field of a strong XFEL pulse (cf. rate equation codes like Ref. [47]), we consider two levels of interest $(|e\rangle,|g\rangle)$ under the influence of several incoherent processes. A general scenario is outlined in Fig. 1, including the following effects: (i) pumping of the ground and excited levels with rates $r_{g}, r_{e}$. Examples of pumping include photoionization by an XFEL pulse [8-10,12] and Auger decay [48-50]; (ii) nonradiative transitions from $|e\rangle$ to $|g\rangle$ with a rate $\gamma_{n}$; (iii) decoherence between levels $|e\rangle,|g\rangle$ with a rate $q$, as caused - for example-by collisions $[49,50]$; and (iv) depletion of the levels with rates $\gamma_{g}, \gamma_{e}$, e.g., through further photoionization by the XFEL pump or decay processes like Auger decay; besides these processes, radiative decay from $|e\rangle$ to $|g\rangle$ and induced transitions between $|e\rangle$ and $|g\rangle$ are already modeled within our current approach. The quantity that determines the coupling between the levels and the emitted radiation is $\Gamma_{\mathrm{sp}}$. Note that the rates $r_{g}, r_{e}, q, \gamma_{g}, \gamma_{e}$ may be conditioned by the external XFEL pulse or the local atomic density, hence they can depend on position and time explicitly.

\section{B. Description of incoherent processes in terms of Lindblad superoperators}

Incoherent processes are modeled as an interaction of the system with an appropriate reservoir. We assume that the reservoirs for all processes and for all atoms are independent and furthermore that these incoherent processes can be described in the Markovian approximation. In this way, we can eliminate the reservoir's variables and describe the transitions between the levels of interest with the help of a master equation [51,52],

$$
\frac{\partial \hat{\rho}^{(a)}}{\partial t}=\frac{1}{i \hbar}\left[\hat{H}, \hat{\rho}^{(a)}\right]+L^{(a)}\left\{\hat{\rho}^{(a)}\right\} .
$$

Here, $L^{(a)}\left\{\hat{\rho}^{(a)}\right\}$ denotes the nonunitary part of the evolution, which describes transitions due to the incoherent process of interest. It can be considered as a Lindblad superoperator acting on the density matrix. For the processes depicted in Fig. 1 we use Lindblad superoperators of the following form [52]:

$$
\begin{aligned}
L_{r, l}^{(a)}\left\{\hat{\rho}^{(a)}\right\}= & \frac{1}{2} \tilde{r}_{l}\left(z_{a}, \tau\right)\left[2 \hat{\sigma}_{+, x l}^{(a)} \hat{\rho}^{(a)} \hat{\sigma}_{-, x l}^{(a)}\right. \\
& \left.-\hat{\sigma}_{-, x l}^{(a)} \hat{\sigma}_{+, x l}^{(a)} \hat{\rho}^{(a)}-\hat{\rho}^{(a)} \hat{\sigma}_{-, x l}^{(a)} \hat{\sigma}_{+, x l}^{(a)}\right]
\end{aligned}
$$

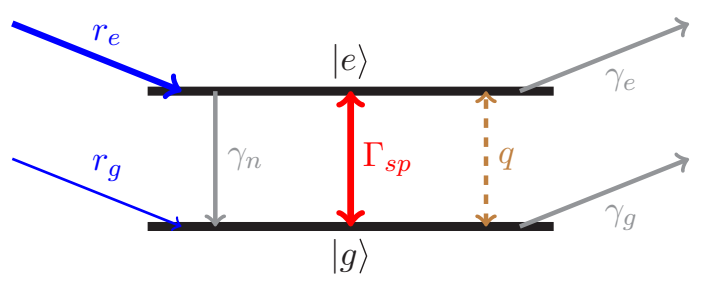

FIG. 1. Sketch of the two levels of interest subject to pumping with rates $r_{e}, r_{g}$, nonradiative decay with a rate $\gamma_{n}$, depletion with rates $\gamma_{g}, \gamma_{e}$, decoherence with a rate $q$, and radiative transitions, which are treated self-consistently within our approach-the typical value determining the evolution rate being $\Gamma_{\mathrm{sp}}$. 


$$
\begin{gathered}
L_{\gamma, l}^{(a)}\left\{\hat{\rho}^{(a)}\right\}= \\
\frac{1}{2} \gamma_{l}\left(z_{a}, \tau\right)\left[2 \hat{\sigma}_{-, x l}^{(a)} \hat{\rho}^{(a)} \hat{\sigma}_{+, x l}^{(a)}-\hat{\sigma}_{+, x l}^{(a)} \hat{\sigma}_{-, x l}^{(a)} \hat{\rho}^{(a)}\right. \\
\left.-\hat{\rho}^{(a)} \hat{\sigma}_{+, x l}^{(a)} \hat{\sigma}_{-, x l}^{(a)}\right], \\
L_{n}^{(a)}\left\{\hat{\rho}^{(a)}\right\}=\frac{1}{2} \gamma_{n}\left[2 \hat{\sigma}_{-}^{(a)} \hat{\rho}^{(a)} \hat{\sigma}_{+}^{(a)}-\hat{\sigma}_{+}^{(a)} \hat{\sigma}_{-}^{(a)} \hat{\rho}^{(a)}-\hat{\rho}^{(a)} \hat{\sigma}_{+}^{(a)} \hat{\sigma}_{-}^{(a)}\right], \\
L_{q}^{(a)}\left\{\hat{\rho}^{(a)}\right\}=\frac{1}{4} q\left(z_{a}, \tau\right)\left[4 \hat{\sigma}_{z}^{(a)} \hat{\rho}^{(a)} \hat{\sigma}_{z}^{(a)}-\hat{\rho}^{(a)}\right] .
\end{gathered}
$$

With $l=e, g$, Eq. (29) corresponds to pumping and Eq. (30) to depletion of level $|l\rangle, \tilde{r}_{l}$ denotes the pumping rate normalized to the occupation of the state, from which pumping takes place (see details in Appendix D). Analogously, $\gamma_{l}$ is the depletion rate. Nonradiative decay with a rate $\gamma_{n}$ is described by Eq. (31) and decoherence with rate $q$ is described by Eq. (32). The operators $\hat{\sigma}_{ \pm, x l}^{(a)}$ account for transitions of atom $a$ between an auxiliary level $|x\rangle$ and level $|l\rangle$ :

$$
\hat{\sigma}_{+, x l}=|l\rangle\left\langle x\left|, \hat{\sigma}_{-, x l}=\right| x\right\rangle\langle l| .
$$

Finally, the total Lindblad superoperator in Eq. (28) becomes

$$
L^{(a)}=L_{r, e}^{(a)}+L_{r, g}^{(a)}+L_{n}^{(a)}+L_{q}^{(a)}+L_{\gamma, e}^{(a)}+L_{\gamma, g}^{(a)} .
$$

The modification of Eqs. (14), (18), and (22) due to Eq. (34) can be derived with the help of quantum Einstein relations [39] (see the derivation in Appendix D for details). In Sec. III D, we give a summary of the ensuing changes.

\section{Absorption of the emitted field}

Another important process that should be taken into account for a realistic treatment of collective spontaneous emission in $\mathrm{x}$-ray (XUV) domain is nonresonant absorption of the emitted radiation. It can be accounted for by adding an imaginary part to the field wave vector: $\vec{k} \rightarrow \vec{k}+i \frac{\kappa}{2} \vec{k}$, where $\kappa$ is absorption coefficient. Incorporation of this factor in Eq. (7) after performing the 1D approximation modifies Eq. (10) to

$$
\hat{A}_{+}^{(a)}(\tau)=-\frac{i \Delta o e p^{*} \Omega}{16 \pi^{2} \epsilon_{0} m c^{2}} e^{-\frac{\kappa}{2}\left(z_{a}-z_{b}\right)} \sum_{b<a} \hat{\sigma}_{-}^{(b)}(\tau) .
$$

\section{Equations of motion for atomic system and field radiation}

Collecting the results from Appendix D and adding the absorption factor that appeared in Eq. (35) we arrive at the following system of equations:

$$
\begin{gathered}
\quad \frac{\partial \rho_{e e}(z, \tau)}{\partial \tau}=r_{e}(z, \tau)-\Gamma_{e e}(z, \tau) \rho_{e e}(z, \tau)-\frac{3 \Delta o}{8 \pi} \Gamma_{\mathrm{sp}} n \int_{0}^{z} d z^{\prime} \mathcal{A}\left(z, z^{\prime}\right) S\left(z, z^{\prime}, \tau\right) \\
\frac{\partial \rho_{g g}(z, \tau)}{\partial \tau}=r_{g}(z, \tau)+\left(\Gamma_{\mathrm{sp}}+\gamma_{n}\right) \rho_{e e}(z, \tau)-\gamma_{g}(z, \tau) \rho_{g g}(z, \tau)+\frac{3 \Delta o}{8 \pi} \Gamma_{\mathrm{sp}} n \int_{0}^{z} d z^{\prime} \mathcal{A}\left(z, z^{\prime}\right) S\left(z, z^{\prime}, \tau\right), \\
\frac{\partial S\left(z_{1}, z_{2}, \tau\right)}{\partial \tau}=-\frac{1}{2}\left[\Gamma\left(z_{1}, \tau\right)+\Gamma\left(z_{2}, \tau\right)\right] S\left(z_{1}, z_{2}, \tau\right)+\frac{3 \Delta o}{16 \pi} \Gamma_{\mathrm{sp}} n\left[\rho_{\mathrm{inv}}\left(z_{1}, \tau\right) \int_{0}^{z_{1}} d z_{1}^{\prime} \mathcal{A}\left(z_{1}, z_{1}^{\prime}\right) S\left(z_{1}^{\prime}, z_{2}, \tau\right)\right. \\
\left.\quad+\rho_{\mathrm{inv}}\left(z_{2}, \tau\right) \int_{0}^{z_{2}} d z_{2}^{\prime} \mathcal{A}\left(z_{2}, z_{2}^{\prime}\right) S\left(z_{1}, z_{2}^{\prime}, \tau\right)\right]+\frac{3 \Delta o}{16 \pi} \Gamma_{\mathrm{sp}}\left[\rho_{\mathrm{inv}}\left(z_{1}, \tau\right) \rho_{e e}\left(z_{2}, \tau\right) \mathcal{A}\left(z_{1}, z_{2}\right) \Theta\left(z_{1}-z_{2}\right)\right. \\
\left.\quad+\rho_{\mathrm{inv}}\left(z_{2}, \tau\right) \rho_{e e}\left(z_{1}, \tau\right) \mathcal{A}\left(z_{2}, z_{1}\right) \Theta\left(z_{2}-z_{1}\right)\right] \\
\frac{\partial G\left(z, \tau_{1}, \tau_{2}\right)}{\partial z}=-\kappa(z) G\left(z, \tau_{1}, \tau_{2}\right)+\frac{3 \Delta o}{16 \pi} \Gamma_{\mathrm{sp}} n\left[\int_{0}^{\tau_{1}} d \tau_{1}^{\prime} e^{i \Omega\left(\tau_{1}-\tau_{1}^{\prime}\right)} \mathcal{D}\left(z, \tau_{1}, \tau_{1}^{\prime}\right) \rho_{\mathrm{inv}}\left(z, \tau_{1}^{\prime}\right) G\left(z, \tau_{1}^{\prime}, \tau_{2}\right)\right. \\
\left.+\int_{0}^{\tau_{2}} d \tau_{2}^{\prime} e^{-i \Omega\left(\tau_{2}-\tau_{2}^{\prime}\right)} \mathcal{D}\left(z, \tau_{2}, \tau_{2}^{\prime}\right) \rho_{\mathrm{inv}}\left(z, \tau_{2}^{\prime}\right) G\left(z, \tau_{1}, \tau_{2}^{\prime}\right)\right]+\frac{3 \Delta o}{32 \pi \lambda^{2}} \Gamma_{\mathrm{sp}} n e^{i \Omega\left(\tau_{1}-\tau_{2}\right)}\left\{\mathcal{D}\left(z, \tau_{1}, 0\right) \mathcal{D}\left(z, \tau_{2}, 0\right) \rho_{e e}(z, 0)\right. \\
\left.+\int_{0}^{\min \tau_{1}, \tau_{2}} d \tau^{\prime} \mathcal{D}\left(z, \tau_{1}, \tau^{\prime}\right) \mathcal{D}\left(z, \tau_{2}, \tau^{\prime}\right)\left[r_{e}\left(z, \tau^{\prime}\right)+\left(\Gamma\left(z, \tau^{\prime}\right)-\Gamma_{e e}\left(z, \tau^{\prime}\right)\right) \rho_{e e}\left(z, \tau^{\prime}\right)\right]\right\}
\end{gathered}
$$

Here, decay rates $\Gamma\left(z, \tau^{\prime}\right), \Gamma_{e e}\left(z, \tau^{\prime}\right)$ have been introduced (see Appendix D for derivations) and read

$$
\Gamma(z, \tau)=\Gamma_{\mathrm{sp}}+\gamma_{n}+q(z, \tau)+\gamma_{e}(z, \tau)+\gamma_{g}(z, \tau), \quad \Gamma_{e e}(z, \tau)=\Gamma_{\mathrm{sp}}+\gamma_{e}(z, \tau)+\gamma_{n}
$$

The term $\mathcal{A}$ is responsible for field absorption and is assumed to be position dependent; the term $\mathcal{D}$ is responsible for decoherence due to spontaneous decay and other incoherent processes. The explicit expressions read

$$
\mathcal{A}\left(z_{2}, z_{1}\right)=e^{-\frac{1}{2} \int_{z_{1}}^{z_{2}} d z^{\prime} \kappa\left(z^{\prime}\right)}, \quad \mathcal{D}\left(z, \tau_{2}, \tau_{1}\right)=e^{-\frac{1}{2} \int_{\tau_{1}}^{\tau_{2}} d \tau^{\prime} \Gamma\left(z, \tau^{\prime}\right)} .
$$

The intensity of the emitted field expressed in terms of atomic variables is modified to

$$
I(z, \tau)=\frac{3 \Delta o}{32 \pi \lambda^{2}} \Gamma_{\mathrm{sp}}\left[n^{2} \int_{0}^{z} d z_{1}^{\prime} \int_{0}^{z} d z_{2}^{\prime} \mathcal{A}\left(z, z_{1}^{\prime}\right) \mathcal{A}\left(z, z_{2}^{\prime}\right) S\left(z_{1}^{\prime}, z_{2}^{\prime}, \tau\right)+n \int_{0}^{z} d z^{\prime} \mathcal{A}^{2}\left(z, z^{\prime}\right) \rho_{e e}\left(z^{\prime}, \tau\right)\right] .
$$

\section{DISCUSSION AND NUMERICAL EXAMPLES}

The system of Eqs. (36)-(39) provides a unified treatment of incoherent and coherent processes taking place during the collective spontaneous emission. Namely, as one limiting case it allows for the description of incoherent spontaneous emission by 
individual atoms. In the opposite limit it includes the propagation of coherent electromagnetic pulses in a medium of two-level atoms, which can be reformulated in the terms of semiclassical Maxwell-Bloch equations [39]. Let us consider each of limiting cases in more detail.

\section{A. Spontaneous emission}

An important limiting case that should be reproduced by our approach is the number of spontaneously emitted photons per unit time into the solid angle $\Delta o$. A reference for this quantity can be calculated based on the usual quantum optical formalism; see Refs. [39,41]. This employs the expression of the emitted field in terms of atomic transition operators, see [39] Ch. 10A, and results in

$$
\begin{aligned}
\frac{d}{d \tau} & N_{\mathrm{ph}, \mathrm{sp}}(\tau, \theta=\pi / 2) \\
& =\frac{2 \epsilon_{0} c}{\hbar \omega}\left\langle\hat{\vec{E}}_{-}\left(\tau, \theta=\frac{\pi}{2}\right) \hat{\vec{E}}_{+}\left(\tau, \theta=\frac{\pi}{2}\right)\right\rangle n z r^{2} \Delta o \\
& =\frac{3}{8 \pi} \Gamma_{\mathrm{sp}} \rho_{e e}(\tau) n z \Delta o .
\end{aligned}
$$

Here, we have used the value of the spontaneous emission rate Eq. (A9); $r$ is the distance from the atoms to a remote observation point; $\theta$ is the angle between the emission direction and the vector of the atomic dipole moment, which we consider to be $\theta=\pi / 2$ for forward emission; $z$ is the length of the system.

Within our approach, i.e., Eqs. (36)-(39) one can obtain spontaneous emission rate if one neglects correlation between atomic coherences. Then from Eq. (42) according to the used convention for intensity Eq. (20) one obtains

$$
\begin{gathered}
\frac{d N_{\mathrm{ph}, \mathrm{sp}}(\tau)}{d \tau}=I(z, \tau) \Delta o \pi R^{2}=\frac{3}{8 \pi} \Gamma_{\mathrm{sp}} \rho_{e e}(\tau) n z \Delta o \xi, \\
\xi=\frac{\Delta o \pi R^{2}}{4 \lambda^{2}} .
\end{gathered}
$$

This expression is in agreement with the exact result of Eq. (43) up to a factor $\xi$ that appears due to the 1D approximation Eq. (8). The actual value of $\Delta o$ is subject to the detail of the approximation, however, the typical estimate based on diffraction $\Delta o \sim \lambda^{2} / R^{2}$ is in agreement with $\xi \sim 1$.

The spectral properties could be obtained directly from the expression for the two-time correlation function Eq. (26). In the case of spontaneous emission one can drop the term involving the field correlation function on the right-hand side of Eq. (26) and obtain the time dependence of the form $e^{i \Omega\left(\tau_{1}-\tau_{2}\right)} e^{-\frac{\Gamma_{\mathrm{sp}}}{2}\left(\tau_{1}+\tau_{2}\right)}$. This form agrees with the exact result for a single two-level atom, see, e.g., Ref. [41], Eq. (10.14b), and results in a Lorentzian spectrum.

\section{B. Maxwell-Bloch equations}

Under the conditions that a large number of photons has been emitted and essential correlations between atomic coherences have been built up, one can expect that the behavior of the system can be described semi-classically. This is typically done by means of Maxwell-Bloch equations, which are widely used for the description of superradiance in the optical domain $[18,20]$ as well as for the description of collective spontaneous emission in the x-ray (XUV) domain [12,23]. We shall subsequently outline the connection between our correlation function approach Eqs. (36)-(39) and MaxwellBloch equations.

A crude way to obtain semiclassical equations is to replace operators by $c$-numbers in the Heisenberg equations of motion. If one carried out this replacement in Eqs. (10)-(12), one would obtain equations that are similar to Eqs. (36)-(39) except for the last term in both Eqs. (38) and (39). These last terms are responsible for spontaneous emission. During the derivation of them, we have essentially used quantum properties of operators (3)-in particular $\left\langle\hat{\sigma}_{+}(\tau) \hat{\sigma}_{-}(\tau)\right\rangle=\rho_{e e}(\tau)$. This property is crucial for the correct description of spontaneous emission and approaches based on the semi-classical relation $\left\langle\hat{\sigma}_{+}(\tau) \hat{\sigma}_{-}(\tau)\right\rangle=\sigma_{+}(\tau) \sigma_{-}(\tau)$ (sometimes referred to as neoclassical theories) cannot reproduce the associated exponential decay [41].

If the dynamics is dominated by the emitted field, we can, however, neglect the spontaneous terms in Eqs. (36)-(39) safely. Using this approximation, Eqs. (36)-(39) reduce to Maxwell-Bloch equations, if we also assume the correlation functions to factorize:

$$
\begin{gathered}
S\left(z_{1}, z_{2}, \tau\right)=\rho_{g e}\left(z_{1}, \tau\right) \rho_{e g}\left(z_{2}, \tau\right), \\
G\left(z, \tau_{1}, \tau_{2}\right)=\frac{2 \epsilon_{0} c}{\Delta o \hbar \Omega} e^{i \Omega\left(\tau_{1}-\tau_{2}\right)} \mathcal{E}_{-}\left(z, \tau_{1}\right) \mathcal{E}_{+}\left(z, \tau_{2}\right) .
\end{gathered}
$$

Here, $\rho_{g e}, \rho_{e g}$ are off-diagonal elements of the density matrix (coherences). In Eq. (47), we have expressed the field correlation function in terms of the slowly varying electric field envelopes $\mathcal{E}_{ \pm}(z, \tau)$ to be compliant with typical Maxwell-Bloch formulations [18,23]. Altogether, we obtain from Eqs. (36)-(39) equations for the density matrix elements and the field (see details in Appendix E):

$$
\begin{gathered}
\frac{\partial \rho_{e e}(z, \tau)}{\partial \tau}=r_{e}(z, \tau)-\Gamma_{e e}(z, \tau) \rho_{e e}(z, \tau) \\
-\frac{2 i \mu}{\hbar} \mathcal{E}_{-}(z, \tau) \rho_{e g}(z, \tau), \\
\frac{\partial \rho_{g g}(z, \tau)}{\partial \tau}=r_{g}(z, \tau)+\left(\Gamma_{\mathrm{sp}}+\gamma_{n}\right) \rho_{e e}(z, \tau) \\
-\gamma_{g}(z, \tau) \rho_{g g}(z, \tau)-\frac{2 i \mu}{\hbar} \mathcal{E}_{+}(z, \tau) \rho_{g e}(z, \tau), \\
\frac{\partial \rho_{g e}(z, \tau)}{\partial \tau}=-\frac{\Gamma(z, \tau)}{2} \rho_{g e}(z, \tau)+\frac{i \mu}{\hbar} \rho_{\text {inv }}(z, \tau) \mathcal{E}_{-}(z, \tau), \\
\frac{\partial \mathcal{E}_{+}(z, \tau)}{\partial z}=-\frac{\kappa(z)}{2} \mathcal{E}_{+}(z, \tau)+\frac{i \Omega}{2 \epsilon_{0} c} \mu n_{3} \rho_{e g}(z, \tau) .
\end{gathered}
$$

This system of equations agrees with the typically used Maxwell-Bloch system of equations [18]. As stated before, however, it shows essential weakness in the treatment of collective spontaneous emission: Assuming the initial absence of fields and coherences, neither of them will appear later on according to Eqs. (50) and (51). This is an immediate consequence of neglecting the spontaneous emission terms in the transition from Eqs. (36)-(39) to Eqs. (48)-(51). There 

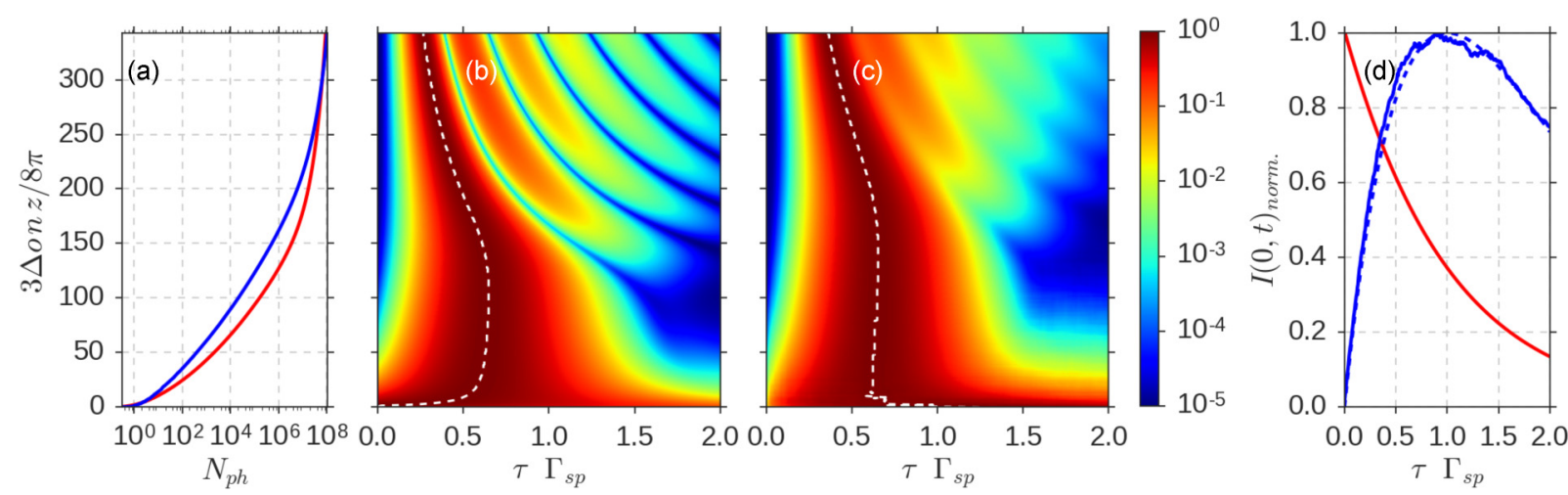

FIG. 2. (a) Simulation of emitted photon number versus system length (red line, correlation function calculation; blue line, Maxwell-Bloch approach); (b) simulation of temporal intensity profiles for systems of different length $z$, calculation according to the correlation function Eqs. (24), (25), and (27), dashed white line shows the position of the intensity maximum; (c) the same figure obtained from the Maxwell-Bloch Eqs. (48)-(51) with noise terms Eqs. (52) and (F5), averaged over 100 realizations; (d) section of the same data taken at $z=0$, the correlation function calculation (red curve) coincides with $e^{-\Gamma_{\mathrm{pp}} \tau}$, while the Maxwell-Bloch result averaged over 1000 realizations (blue curve) approaches $\Gamma_{\mathrm{sp}} \tau e^{-\Gamma_{\mathrm{sp}} \tau} e$ (dashed blue line).

are a number of semiphenomenological ways of reintroducing spontaneous emission into the Maxwell-Bloch Eqs. (48)-(51). For applications connected with spontaneous emission in the $\mathrm{X}$-ray (XUV) domain, the most widespread approach is to add random noise term on the right-hand side of the equations for the coherences [10,12,22-24,26,53,54]. In the frame of this approach, the noise terms are assumed to have the following properties:

$$
\begin{aligned}
\left\langle s_{ \pm}(z, t)\right\rangle & =0, \quad\left\langle s_{+}\left(z_{1}, t_{1}\right) s_{-}\left(z_{2}, t_{2}\right)\right\rangle \\
& =\rho_{e e}\left(z_{1}, t_{1}\right) F \delta\left(z_{1}-z_{2}\right) \delta\left(t_{1}-t_{2}\right),
\end{aligned}
$$

where $\langle\ldots\rangle$ means statistical average over multiple realizations. The coefficient $F$ is chosen to fit the value of the spontaneous emission. This and the resulting temporal profile of the spontaneous emission were recently discussed in detail in Ref. [55]. As it was shown there, this kind of noise model does not reproduce the expected exponential decay. As an example, we consider simplified case without incoherent processes and complete initial excitation in Appendix F. For this scenario, the Maxwell-Bloch equations with noise terms Eqs. (52) predict a temporal behavior as $\Gamma_{\mathrm{sp}} t e^{-\Gamma_{\mathrm{sp}} t}$, which can be tolerable for cases with a short decoherence time. However, for any choice of parameters, the Maxwell-Bloch equations with noise terms Eqs. (52) will produce an unrealistic, timedelayed peak of the spontaneous emission.

A visual comparison of the temporal intensity profiles based on the Maxwell-Bloch equations (using the noise term correlation factor derived in Appendix F) with calculations according to our correlation function Eqs. (24), (25), and (27) is given in Fig. 2. Here, a case of instantaneous swept pumping is considered, namely, a complete population inversion is assumed at $\tau=0$. In Figs. 2(b) and 2(c), the radiated intensity is plotted versus retarded time $\tau$ and the length of the medium $z$. For each value of $z$, the intensity is normalized to the maximal value. Figure 2(b) shows results based on Eqs. (24), (25), and (27), whereas Fig. 2(c) is obtained from the Maxwell-Bloch approach. From Eqs. (24), (25), and (27), one can see that if one uses the dimensionless time $\tau \Gamma_{\mathrm{sp}}$ and the dimensionless length $3 \Delta o n z / 8 \pi$, then the only parameter that defines the temporal shape of the intensity profile of the system is the solid angle $\Delta o$. The value $\Delta o=4 \times 10^{-6}$ was used for the simulations presented in Fig. 2.

At short length of the medium, the stimulated emission can be neglected, hence the temporal evolution of the intensity should have the form of spontaneous decay $e^{-\Gamma_{\mathrm{sp}} \tau}$. Figure 2(d) shows sections of Figs. 2(b) and 2(c) close to $z=0$. As we discussed above, our approach shows the expected exponential decay behavior, while the Maxwell-Bloch approach follows a time dependence like $\Gamma_{\mathrm{sp}} t e^{-\Gamma_{\mathrm{sp}} t}$. The calculated temporal profile shows noisy behavior even after averaging over 1000 realizations. With increasing system length $z$, the stimulated emission results in exponential-like growth of the emitted photons number, see Fig. 2(a). During this process, the temporal intensity profile transforms from exponential decay (peaked at $\tau=0$ ) to a profile showing a delayed peak; see Fig. 2(b). In the frame of the Maxwell-Bloch approach, the temporal profile always shows a delayed peak, even at infinitesimally small $z$. The position of this peak is strongly influenced by noise at small $z$ and stays approximately constant up to the onset of saturation; see Fig. 2(c). After the saturation sets in (after $3 \Delta o n z / 8 \pi \sim 150$ ), oscillatory behavior (ringing) appears. These oscillations are smeared in the MaxwellBloch approach due to the finite number of realizations. In both models, the period of the oscillations decreases with increasing system length $z$ and the position of the peak tends to shorter times.

\section{Numerical examples}

The correlation function Eqs. (36)-(39) can be used to model various realistic systems, provided the $1 \mathrm{D}$ approximation is applicable. As a first example let us consider evolution induced by an XFEL pulse in Neon gas. Calculations for Fig. 3 were done at parameters corresponding to experiment described in Ref. [8] and aim to compare with Maxwell-Bloch calculations performed for the same system in Ref. [12]. In particular, the Neon gas cell with density $1.6 \times 10^{19} \mathrm{~cm}^{-3}$ and length $15 \mathrm{~mm}$ is considered to be irradiated by a an XFEL pulse with photon energy $880 \mathrm{eV}$, containing $2 \times 10^{12}$ photons 

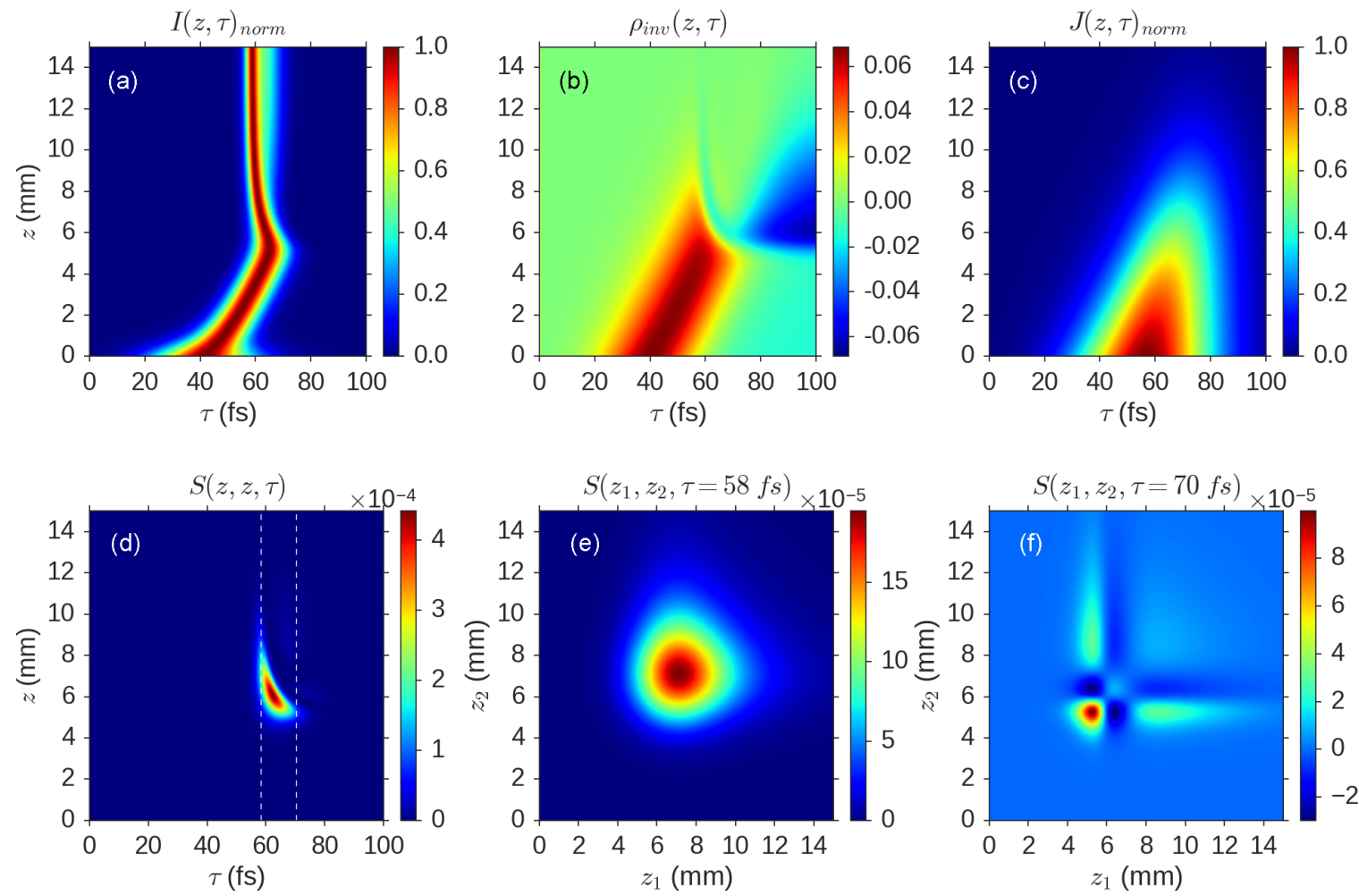

FIG. 3. Evolution of collective spontaneous emission induced by an XFEL pump in Ne gas. (a) Profile of emitted superfluorescence intensity normalized to 1 for each $z$ value; (b) population inversion; (c) profile of the XFEL pump pulse normalized to maximal value; (d) diagonal part of correlation function of atomic coherences; (e), (f) values of correlation function of atomic coherences taken at time moments 55 and 70 fs.

within 40 fs FWHM Gaussian temporal profile, focusing with $2 \mu \mathrm{m}$ radius is assumed. The photoionization of $1 s$ electrons with crossection $0.3 \mathrm{Mb}$ results in population inversion between $1 s^{1} 2 s^{2} 2 p^{6}$ and $1 s^{2} 2 s^{2} 2 p^{5}$ states. The transition between these states results in emission at $1.46 \mathrm{~nm}$ wavelength, the spontaneous lifetime is $160 \mathrm{fs}$, the competing process is Auger decay with 2.4 fs decay time.

In Fig. 3(a) the temporal intensity profile normalized at each value of $z$ [similar to Figs. 2(b) and 2(c)] is shown. The temporal position of the peak at small $z$ values is determined by excitation of atoms. The excitation is conditioned by the pump pulse, its spatiotemporal evolution due to absorption is shown in Fig. 3(c). Figure 3(b) shows the population inversion. Before saturation (at $\sim 6 \mathrm{~mm}$ ) the evolution of population inversion is mainly determined by the pump, the peak of intensity comes shortly after the peak of population inversion. After saturation, oscillatory behavior can be seen in the population inversion, the peak of intensity temporal profile shifts to shorter times. The evolution of observables presented in Figs. 3(a)-3(c) can be compared directly to that in Figs. 5(a)-5(c) in Ref. [12]. To have qualitative agreement for propagation distances above $\sim 2 \mathrm{~mm}$ the value of solid angle $\Delta o$ was taken as $2 \pi R^{2} / L^{2}$. Despite of qualitative agreement for larger propagation distances, the Maxwell-Bloch calculation of intensity profile below $\sim 2 \mathrm{~mm}$ of propagation show fluctuating behavior due to stochastic noise terms. As we have seen in Fig. 2, the Maxwell-Bloch approach with noise term does not describe the initial amplification stage adequately. The analytical treatment of this stage for the considered system is complicated due to pumping and Auger processes taking place on the same timescale as the emission. Therefore, the application of analytical Bessel-function-based solutions known for instantaneously pumped systems $[18,32,33]$ is not possible. Under these conditions, the presented approach is the method of choice to trace the evolution from spontaneous emission to amplified emission and superfluorescence.

The correlation function of atomic coherences $S\left(z_{1}, z_{2}, \tau\right)$ can be considered as source of stimulated emission, see Eq. (42). Several sections of this function are shown in Figs. 3(d)-3(f). Specifically, Fig. 3(d) shows the correlation function of the atomic coherence at infinitesimally close points $z_{1}=z_{2}=z$ at retarded time moments $\tau$. Its peak at $\sim 60 \mathrm{fs}, \sim 6 \mathrm{~mm}$ corresponds to the onset of oscillations in the population inversion [see Fig. 3(b)] and saturation. The twopoint correlation function $S\left(z_{1}, z_{2}, \tau\right)$ for a fixed time moment $\tau$ represents purely spatial correlation of the polarization of the medium, and is represented for times $\tau=55$ fs and $\tau=70 \mathrm{fs}$ in Figs. 3(e) and 3(f), i.e., for times before and after the maximum of $S(z, z, \tau)$. Before onset of saturation ( $\tau=55 \mathrm{fs}$ ) the correlation function $S(z, z, \tau)$ shows a single maximum. After saturation, several local maxima and minima (anti-correlation) are visible. Beyond saturation, the population of the excited state drops below the population of the ground state (negative population inversion) in some parts of the medium. This results in the absorption of the emitted field and corresponds to negative values of the correlation function of atomic coherences, see Fig. 3(f), while other parts of the sample show positive correlation (emission). This partitioning 

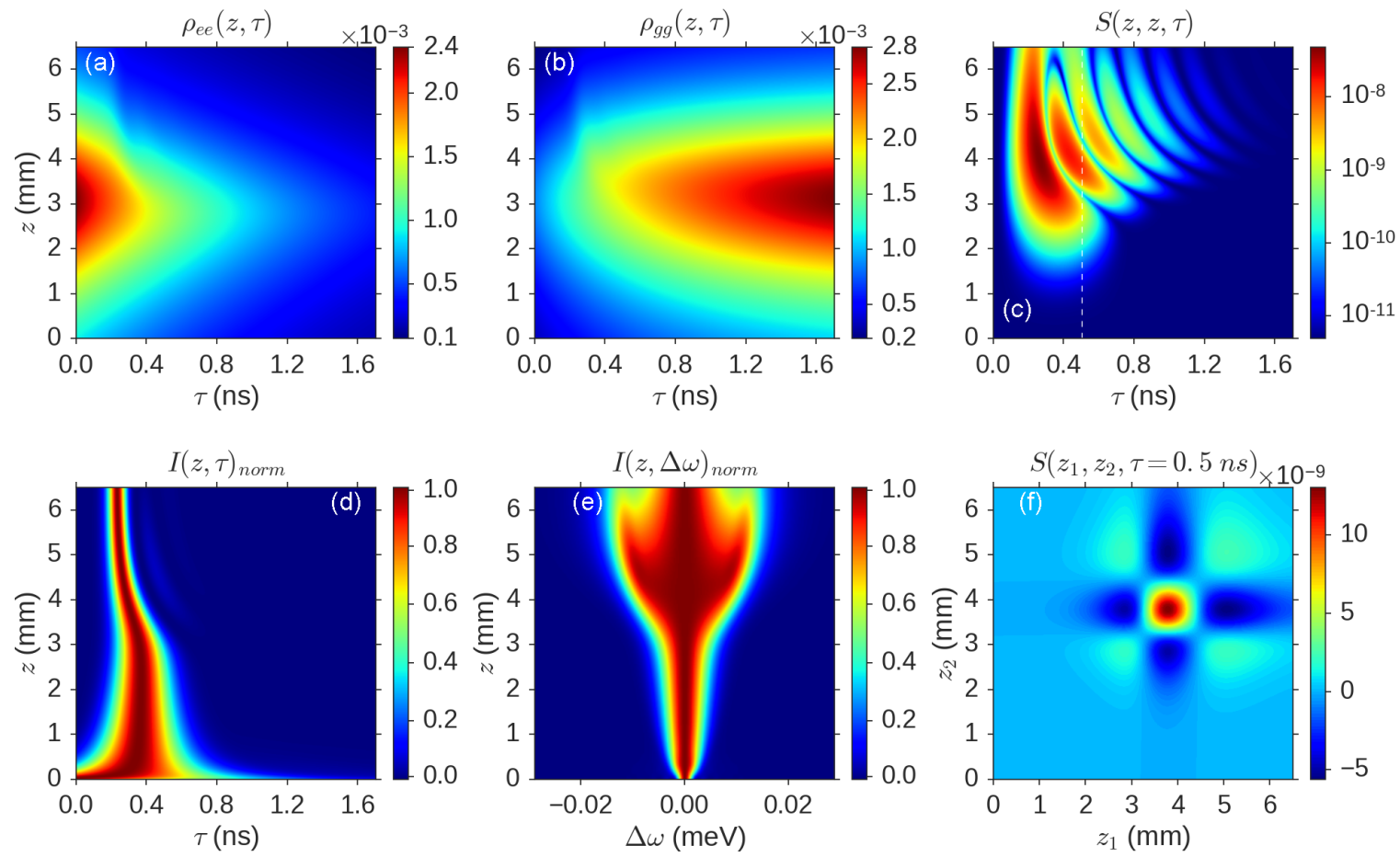

FIG. 4. Evolution of collective spontaneous emission induced by an XFEL pump in Xe gas. (a), (b) Evolution of the excited and ground state populations; (c) temporal evolution of the correlation function of the atomic coherences taken at close spatial points $z_{1}=z_{2}=z$; (d) profile of emitted superfluorescence intensity normalized to 1 for each $z$ value; (e) spatial evolution of emitted radiation spectrum, normalized to 1 for each $z$ value; (f) correlation function of the atomic coherences at the time moment $\tau=0.5 \mathrm{~ns}$.

of the sample into positively and negatively correlated parts is a direct manifestation of the temporal ringing in the spatial domain.

As another example, we consider collective spontaneous emission from Xe atoms pumped by a XFEL pulse at a mean photon energy of $73 \mathrm{eV}$ corresponding to conditions of a recent experiment that was conducted at the soft $\mathrm{x}$-ray freeelectron laser FLASH [56]. Namely, following parameters were used: pump photon energy is $73 \mathrm{eV}$, pump pulse energy is $50 \mu \mathrm{J}$, emitted radiation wavelength is $65 \mathrm{~nm}$, spontaneous life time corresponding to transition from $|e\rangle$ to $|g\rangle$ level is 1 ns, Auger life time is $6 \mathrm{fs}$, Auger branching ratios are taken as 0.021 for $|e\rangle$ level and 0.0075 for $|g\rangle$ level, pump absorption cross section is $5.2 \mathrm{Mb}$, emitted field absorption cross section is $60 \mathrm{Mb}$, gas pressure is 7 mbar, for pump a Gaussian temporal shape is assumed with 80 fs FWHM. To account for the spatial pulse profile of a focused beam, we modeled the intensity as a Gaussian beam with $2.0 \mathrm{~mm}$ Rayleigh range and $61 \mu \mathrm{m}$ waist size. The population inversion in this system is created by Auger decay of a $4 d$ hole state, which is in turn created by photoionization due to the FEL pump [49]. In terms of Eqs. (36)-(39), this scheme corresponds to the inclusion of the following rates:

$$
\begin{gathered}
r_{e, g}(z, \tau)=b_{e, g} \gamma_{A} \rho_{c}(z, \tau), \\
\gamma_{e, g}(z, \tau)=J(z, \tau) \sigma_{i} .
\end{gathered}
$$

Here, $\gamma_{A}$ is the Auger decay rate; $\rho_{c}(z, \tau)$ marks the population of the $4 d$ state, which can be obtained from rate equations for the population of atomic levels; $b_{e, g}$ are the branching ratios of the Auger decay into the levels $|e\rangle$ and $|g\rangle ; J(z, \tau)$ gives the photon flux of the FEL. It is time dependent due to the pulse shape and spatially varying due to the position-dependent beam waist size and absorption; $\sigma_{i}$ denotes the cross section for ionization from the upper and lower level. Inclusion of the dynamics of atomic occupations described by rate equations and the spatial profile of the pump is obligatory for the description of realistic systems. Notably, it would be difficult to perform a similar analysis as in the Appendix F and thereby obtain plausible effective noise terms for Maxwell-Bloch equations under these conditions.

The temporal field intensity profile, Fig. 4(d), shows transformation from spontaneous emission with maxima at $\tau=0$ to peak-shaped temporal profiles that are characteristic for superfluorescence. The peak appears earlier and becomes narrower and more intense with increasing the propagation length.

The field correlation function enables to obtain radiation spectra by means of Fourier transform [39]. For parameters used for modeling Fig. 4, one can see that the occupation of the $|e\rangle$ and $|g\rangle$ levels is of the order of $10^{-3}$, nevertheless after about $3 \mathrm{~mm}$ of propagation the ringing (or Rabi-like behavior) takes place; see Figs. 4(a) and 4(b). It is hardly seen in occupations; however, the correlation of atomic coherences, Fig. 4(c), clearly show the oscillatory behavior in time. This behavior is present in field correlation function as well, it results in spectral broadening clearly seen in Fig. 4(e). This spectral broadening can be used as a marker for the oscillatory superfluorescence regime, and can be particularly helpful when the direct measurement of the temporal pulse profile 
is not possible that is often the case for the x-ray (XUV) domain. At time moments corresponding to ringing, the correlation function of atomic coherences shows oscillatory behavior in space as well, see Fig. 4(f). The number of oppositely correlated regions in space (regions with opposite sign of correlation function of atomic coherences) increases with time, together with decreasing of correlation function of atomic coherences it results in damping of intensity ringing.

\section{CONCLUSIONS}

We have proposed a theoretical approach to collective spontaneous emission that is based on integro-differential equations for the correlation function of atomic coherences and field correlation function. It enables us to obtain the spatiotemporal profiles of level occupations and emitted intensity as well as the spatial profile of the emitted radiation spectrum. Our derivations start from a basic atom-field Hamiltonian; the main approximations are the 1D modeling of the field propagation and the swept pumping configuration. A number of incoherent processes that can take place in realistic systems, such as collisional quenching of coherences, time- and space-dependent pumping and depleting of the levels of interest or nonradiative decay are taken into account systematically by means of the Lindblad formalism. Our approach allows for the treatment of spontaneous emission and coherent phenomena-typically described by Maxwell-Bloch equations - on the same footing. This gives advantage over the widely used approach of modeling collective spontaneous emission in the Maxwell-Bloch equations by means of noise terms. In particular, we show the latter one to be unable to describe the temporal profile of spontaneous emission correctly. As an application of our method, we presented calculations corresponding to a recent experiment [56].

\section{ACKNOWLEDGMENTS}

We gratefully acknowledge a critical reading of the manuscript by D. Krebs.

\section{APPENDIX A: DERIVATION OF HEISENBERG-LANGEVIN EQUATIONS}

From the Hamiltonian Eq. (1) we obtain the following Heisenberg equations:

$$
\begin{gathered}
\frac{d \hat{a}_{\vec{k}, s}}{d t}=-i \omega_{\vec{k}} \hat{a}_{\vec{k}, s}-i g_{\vec{k}, s}^{*} \sum_{a} e^{-i \vec{k} \vec{r}_{a}} \hat{\sigma}_{-}^{(a)}, \\
\frac{d \hat{\sigma}_{-}^{(a)}}{d t}=-i \Omega \hat{\sigma}_{-}^{(a)}+2 i \sum_{\vec{k}, s} g_{\vec{k}, s} e^{i \vec{k} \vec{r}_{a}} \hat{\sigma}_{z}^{(a)} \hat{a}_{\vec{k}, s}, \\
\frac{d \hat{\sigma}_{z}^{(a)}}{d t}=i \sum_{\vec{k}, s}\left(g_{\vec{k}, s}^{*} e^{-i \vec{k} \vec{r}_{a}} \hat{a}_{\vec{k}, s}^{\dagger} \hat{\sigma}_{-}^{(a)}-g_{\vec{k}, s} e^{i \vec{k} \vec{r}_{a}} \hat{\sigma}_{+}^{(a)} \hat{a}_{\vec{k}, s}\right) .
\end{gathered}
$$

Here, we use the normal ordering of operators: on the left side come operators $\hat{a}_{\vec{k}, s}^{\dagger}, \hat{\sigma}_{+}^{(a)}$ followed by $\hat{\sigma}_{z}^{(a)}$ and on the right side $\hat{a}_{\vec{k}, s}, \hat{\sigma}_{-}^{(a)}$, see discussions in Refs. [41,57].
We formally integrate the equation for field operators Eq. (A1),

$$
\begin{aligned}
\hat{a}_{\vec{k}, s}(t)= & \hat{a}_{\vec{k}, s}(0) e^{-i \omega_{\vec{k}} t} \\
& -i g_{\vec{k}, s}^{*} \sum_{a} e^{-i \vec{k} \vec{r}_{a}} \int_{0}^{t} d t^{\prime} e^{-i \omega_{\vec{k}}\left(t-t^{\prime}\right)} \hat{\sigma}_{-}^{(a)}\left(t^{\prime}\right),
\end{aligned}
$$

and substitute it into Eq. (A2):

$$
\begin{aligned}
\frac{d \hat{\sigma}_{-}^{(a)}}{d t}= & -i \Omega \hat{\sigma}_{-}^{(a)} \\
& +2 \sum_{b, \vec{k}, s}\left|g_{\vec{k}, s}\right|^{2} \int_{0}^{t} d t^{\prime} e^{i\left[\vec{k}\left(\vec{r}_{a}-\vec{r}_{b}\right)-\omega\left(t-t^{\prime}\right)\right]} \hat{\sigma}_{z}^{(a)}(t) \hat{\sigma}_{-}^{(b)}\left(t^{\prime}\right) \\
& +2 i \sum_{\vec{k}, s} g_{\vec{k}, s} \hat{\sigma}_{z}^{(a)}(t) \hat{a}_{\vec{k}, s}(0) e^{i\left(\vec{k} \vec{r}_{a}-\omega t\right)} .
\end{aligned}
$$

The last term is determined by the vacuum field at the initial time and can be interpreted as a stochastic Langevin term:

$$
\begin{aligned}
& \hat{F}_{-}^{(a)}(t)=2 i \sum_{\vec{k}, s} g_{\vec{k}, s} \hat{\sigma}_{z}^{(a)}(t) \hat{a}_{\vec{k}, s}(0) e^{i\left(\vec{k} \vec{r}_{a}-\omega t\right)}, \\
& \hat{F}_{+}^{(a)}(t)=-2 i \sum_{\vec{k}, s} g_{\vec{k}, s}^{*} \hat{a}_{\vec{k}, s}^{\dagger}(0) \hat{\sigma}_{z}^{(a)}(t) e^{-i\left(\vec{k} \vec{r}_{a}-\omega t\right)} .
\end{aligned}
$$

Here, $\hat{F}_{+}^{(a)}(t)$ corresponds to the analogous term in the equation for $\hat{\sigma}_{+}^{(a)}$. The statistical properties of these terms can be obtained directly from their definition Eq. (A6) taking into account that the field is initially in the vacuum state:

$$
\begin{aligned}
\left\langle\hat{F}_{-}^{(a)}(t)\right\rangle= & \left\langle\hat{F}_{+}^{(a)}(t)\right\rangle=0, \\
\left\langle\hat{F}_{-}^{(a)}(t) \hat{F}_{-}^{(b)}\left(t^{\prime}\right)\right\rangle= & \left\langle\hat{F}_{+}^{(a)}(t) \hat{F}_{+}^{(b)}\left(t^{\prime}\right)\right\rangle=\left\langle\hat{F}_{+}^{(a)}(t) \hat{F}_{-}^{(b)}\left(t^{\prime}\right)\right\rangle=0, \\
\left\langle\hat{F}_{-}^{(a)}(t) \hat{F}_{+}^{(b)}\left(t^{\prime}\right)\right\rangle= & 4\left\langle\hat{\sigma}_{z}^{(a)}(t) \hat{\sigma}_{z}^{(b)}\left(t^{\prime}\right)\right\rangle \\
& \times \sum_{\vec{k}, s}\left|g_{\vec{k}, s}\right|^{2} e^{i\left[\vec{k}\left(\vec{r}_{a}-\vec{r}_{b}\right)-\omega\left(t-t^{\prime}\right)\right]} .
\end{aligned}
$$

The sum over atoms in the second term of Eq. (A5) can be split into a part $b=a$ that describes the action of the field emitted by the atom $a$ on itself, and a part $b \neq a$ that describes the interaction of a given atom $a$ with other atoms in the system. Considering the first term, one can expect the action of the field emitted by atom $a$ onto itself results in spontaneous decay:

$$
\begin{aligned}
&\left.\frac{d \hat{\tilde{\sigma}}_{-}^{(a)}}{d t}\right|_{\mathrm{sp}}= 2 \sum_{\vec{k}, s}\left|g_{\vec{k}, s}\right|^{2} \int_{0}^{t} d t^{\prime} e^{-i(\omega-\Omega)\left(t-t^{\prime}\right)} \hat{\sigma}_{z}^{(a)}(t) \hat{\tilde{\sigma}}_{-}^{(a)}\left(t^{\prime}\right) \\
&= \frac{e^{2}|\vec{p}|^{2}}{3 \pi^{2} m^{2} c^{3} \hbar \epsilon_{0}} \hat{\sigma}_{z}^{(a)}(t) \int_{0}^{t} d t^{\prime} \int_{0}^{\infty} d \omega \omega \hat{\tilde{\sigma}}_{-}^{(a)}\left(t^{\prime}\right) \\
& \times e^{-i(\omega-\Omega)\left(t-t^{\prime}\right)} \simeq-\frac{\Gamma_{\mathrm{sp}}}{2} \hat{\tilde{\sigma}}_{-}^{(a)}(t) \\
& \Gamma_{\mathrm{sp}}=\frac{e^{2} \Omega|\vec{p}|^{2}}{3 \pi \epsilon_{0} \hbar m^{2} c^{3}}
\end{aligned}
$$

Here, we made use of slowly varying function $\tilde{\sigma}: \hat{\sigma}_{-}^{(a)}(t)=$ $\hat{\tilde{\sigma}}_{-}^{(a)}(t) e^{-i \Omega t}$; in the last step we applied the approximation 
typically done in Weisskopf-Wigner approach, that is, we treat the spontaneous emission of a single atom as a Markovian process; $\Gamma_{\mathrm{sp}}$ is the corresponding spontaneous emission rate, where we neglected the Lamb shift. Note that the accepted rule of ordering the operators results in the description of spontaneous emission as an effect of radiation reaction alone. Conversely, other ordering conventions would result in contributions from vacuum fluctuations of the field, see Ref. [57], Ch. 7 for further discussion and references.

Next, we consider the induced part of the second term in Eq. (A5). This is responsible for the interaction of a given atom $a$ with other atoms in the system. It can be rewritten in terms of the vector potential of the electromagnetic field at the position of atom $a$ due to all other atoms in the system:

$$
\begin{aligned}
\left.\frac{d \hat{\sigma}_{-}^{(a)}}{d t}\right|_{\text {ind }}= & \frac{2 i e}{m \hbar c} \hat{\sigma}_{z}^{(a)}(t) \vec{p} \cdot \overrightarrow{\hat{A}}_{+}^{(a)}(t) \\
\overrightarrow{\hat{A}}_{+}^{(a)}(t)= & -\frac{i e c}{16 \pi^{3} m \epsilon_{0}} \int d^{3} \vec{k} \sum_{s} \frac{1}{\omega_{\vec{k}}} \vec{e}_{\vec{k}, s}\left(\vec{e}_{\vec{k}, s}^{*} \cdot \vec{p}^{*}\right) \\
& \times \int_{0}^{t} d t^{\prime} \sum_{b \neq a} e^{i\left(\vec{k}\left(\vec{r}_{a}-\vec{r}_{b}\right)-\omega\left(t-t^{\prime}\right)\right)} \hat{\sigma}_{-}^{(b)}\left(t^{\prime}\right)
\end{aligned}
$$

Combining Eqs. (A8) and (A10) we obtain Eq. (5). Similar steps lead to Eq. (6), the corresponding Langevin term having zero mean value:

$$
\left\langle\hat{F}_{z}^{(a)}(t)\right\rangle=0
$$

\section{APPENDIX B: EQUATION OF MOTION FOR THE PRODUCT OF OPERATORS}

The equation of motion for the product of two operators can be obtained from the Heisenberg-Langevin equations of motion for each of the operators with help of generalized Einstein relations [39]. Namely, if two operators satisfy Langevin equations with $\delta$-correlated noise,

$$
\begin{aligned}
\frac{d \hat{O}_{1}}{d t} & =\hat{V}_{1}(t)+\hat{F}_{1}(t), \quad \frac{d \hat{O}_{2}}{d t}=\hat{V}_{2}(t)+\hat{F}_{2}(t), \\
\left\langle\hat{F}_{1}(t)\right\rangle & =\left\langle\hat{F}_{2}(t)\right\rangle=0,\left\langle\hat{F}_{1}(t) \hat{F}_{2}\left(t^{\prime}\right)\right\rangle=D_{12}(t) \delta\left(t-t^{\prime}\right),
\end{aligned}
$$

then observable $\left\langle\hat{O}_{1}(t) \hat{O}_{2}(t)\right\rangle$ will satisfy (see Ref. [39], Ch. 9):

$$
\frac{d\left\langle\hat{O}_{1}(t) \hat{O}_{2}(t)\right\rangle}{d t}=\left\langle\hat{V}_{1}(t) \hat{O}_{2}(t)\right\rangle+\left\langle\hat{O}_{1}(t) \hat{V}_{2}(t)\right\rangle+D_{12}(t)
$$

Applying this expression to Eq. (11) and its Hermitian conjugate, while taking into account Eq. (A7), we obtain Eq. (16).

\section{APPENDIX C: DERIVATION OF THE PROPAGATION EQUATION FOR THE FIELD CORRELATION FUNCTION}

To obtain a spatial propagation equation for $G^{(a)}\left(\tau_{1}, \tau_{2}\right)$ we consider the difference between the field correlation function at position $z_{a}$ of atom $a$ and neighboring atom $a+1$ at $z_{a+1}$. From Eq. (21) we directly obtain

$$
G^{(a+1)}\left(\tau_{1}, \tau_{2}\right)-G^{(a)}\left(\tau_{1}, \tau_{2}\right)=\frac{3 \Delta o}{32 \pi \lambda^{2}} \Gamma_{\mathrm{sp}}\left[\sum_{b<a}\left\langle\hat{\sigma}_{+}^{(b)}\left(\tau_{1}\right) \hat{\sigma}_{-}^{(a)}\left(\tau_{2}\right)\right\rangle+\sum_{b<a}\left\langle\hat{\sigma}_{+}^{(a)}\left(\tau_{1}\right) \hat{\sigma}_{-}^{(b)}\left(\tau_{2}\right)\right\rangle+\left\langle\hat{\sigma}_{+}^{(a)}\left(\tau_{1}\right) \hat{\sigma}_{-}^{(a)}\left(\tau_{2}\right)\right\rangle\right] .
$$

With the help of Eq. (10), we can express quantities like $\sum_{b<a} \hat{\sigma}_{+}^{(b)}\left(\tau_{1}\right), \sum_{b<a} \hat{\sigma}_{-}^{(b)}\left(\tau_{2}\right)$ in terms of the field and thus find:

$$
G^{(a+1)}\left(\tau_{1}, \tau_{2}\right)-G^{(a)}\left(\tau_{1}, \tau_{2}\right)=\frac{i e}{2 \hbar c m \lambda^{2}}\left[p\left\langle\hat{\sigma}_{+}^{(a)}\left(\tau_{1}\right) \hat{A}_{+}^{(a)}\left(\tau_{2}\right)\right\rangle-p^{*}\left\langle\hat{A}_{-}^{(a)}\left(\tau_{1}\right) \hat{\sigma}_{-}^{(a)}\left(\tau_{2}\right)\right\rangle\right]+\frac{3 \Delta o}{32 \pi \lambda^{2}} \Gamma_{\mathrm{sp}}\left\langle\hat{\sigma}_{+}^{(a)}\left(\tau_{1}\right) \hat{\sigma}_{-}^{(a)}\left(\tau_{2}\right)\right\rangle
$$

Next, we shall express atomic coherences in terms of the field. This can be achieved by formal integration of Eq. (11):

$$
\hat{\sigma}_{-}^{(a)}(\tau)=\int_{0}^{\tau} d \tau^{\prime} e^{-\left(i \Omega+\frac{\Gamma_{\mathrm{sp}}}{2}\right)\left(\tau-\tau^{\prime}\right)}\left[\frac{2 i e p}{\hbar m c} \hat{\sigma}_{z}^{(a)}\left(\tau^{\prime}\right) \hat{A}_{+}^{(a)}\left(\tau^{\prime}\right)+\hat{F}_{-}^{(a)}\left(\tau^{\prime}\right)\right]+e^{-\left(i \Omega+\frac{\Gamma_{\mathrm{sp}}}{2}\right) \tau} \hat{\sigma}_{-}^{(a)}(0) .
$$

Substituting Eq. (C3) into Eq. (C2) we obtain

$$
\begin{aligned}
G^{(a+1)}\left(\tau_{1}, \tau_{2}\right)-G^{(a)}\left(\tau_{1}, \tau_{2}\right)= & \frac{3 \epsilon_{0} \Omega}{4 \pi \hbar c} \Gamma_{\mathrm{sp}}\left[\int_{0}^{\tau_{1}} d \tau_{1}^{\prime} e^{-\left(-i \Omega+\frac{\Gamma_{\mathrm{sp}}}{2}\right)\left(\tau_{1}-\tau_{1}^{\prime}\right)}\left\langle\hat{A}_{-}^{(a)}\left(\tau_{1}^{\prime}\right) \hat{\sigma}_{z}^{(a)}\left(\tau_{1}^{\prime}\right) \hat{A}_{+}^{(a)}\left(\tau_{2}\right)\right\rangle\right. \\
& +\int_{0}^{\tau_{2}} d \tau_{2}^{\prime} e^{-\left(i \Omega+\frac{\Gamma_{\mathrm{sp}}}{2}\right)\left(\tau_{2}-\tau_{2}^{\prime}\right)}\left\langle\hat{A}_{-}^{(a)}\left(\tau_{1}\right) \hat{\sigma}_{z}^{(a)}\left(\tau_{2}^{\prime}\right) \hat{A}_{+}^{(a)}\left(\tau_{2}^{\prime}\right)\right]+\frac{3 \Delta o}{32 \pi \lambda^{2}} \Gamma_{\mathrm{sp}}\left\langle\hat{\sigma}_{+}^{(a)}\left(\tau_{1}\right) \hat{\sigma}_{-}^{(a)}\left(\tau_{2}\right)\right\rangle .
\end{aligned}
$$

Here, we took into account Eq. (A7) as well as $\left\langle\hat{\sigma}_{+}^{(a)}(0) \hat{F}_{-}^{(b)}\left(t^{\prime}\right)\right\rangle=0,\left\langle\hat{F}_{+}^{(a)}(t) \hat{\sigma}_{-}^{(b)}(0)\right\rangle=0,\left\langle\hat{\sigma}_{+}^{(a)}(0) \hat{\sigma}_{-}^{(b)}(0)\right\rangle=0$ for $a \neq b$. Making approximation Eq. (17), which enables us to factor out $\left\langle\hat{\sigma}_{z}^{(a)}(\tau)\right\rangle$, we arrive at

$$
\begin{gathered}
G^{(a+1)}\left(\tau_{1}, \tau_{2}\right)-G^{(a)}\left(\tau_{1}, \tau_{2}\right)=\delta G^{(a)}\left(\tau_{1}, \tau_{2}\right)_{\mathrm{stim}}+\delta G^{(a)}\left(\tau_{1}, \tau_{2}\right)_{\mathrm{sp}}, \\
\delta G^{(a)}\left(\tau_{1}, \tau_{2}\right)_{\mathrm{stim}}=\frac{3 \Delta o}{8 \pi} \Gamma_{\mathrm{sp}}\left[\int_{0}^{\tau_{1}} d \tau_{1}^{\prime} e^{-\left(-i \Omega+\frac{\Gamma_{\mathrm{sp}}}{2}\right)\left(\tau_{1}-\tau_{1}^{\prime}\right)}\left\langle\hat{\sigma}_{z}^{(a)}\left(\tau_{1}^{\prime}\right)\right\rangle G^{(a)}\left(\tau_{1}^{\prime}, \tau_{2}\right)+\int_{0}^{\tau_{2}} d \tau_{2}^{\prime} e^{-\left(i \Omega+\frac{\Gamma_{\mathrm{sp}}}{2}\right)\left(\tau_{2}-\tau_{2}^{\prime}\right)}\left\langle\hat{\sigma}_{z}^{(a)}\left(\tau_{2}^{\prime}\right)\right\rangle G^{(a)}\left(\tau_{1}, \tau_{2}^{\prime}\right)\right], \\
(\mathrm{C} 6) \\
(\mathrm{C} 7) \\
\delta G^{(a)}\left(\tau_{1}, \tau_{2}\right)_{\mathrm{sp}}=\frac{3 \Delta o}{32 \pi \lambda^{2}} \Gamma_{\mathrm{sp}}\left\langle\hat{\sigma}_{+}^{(a)}\left(\tau_{1}\right) \hat{\sigma}_{-}^{(a)}\left(\tau_{2}\right)\right\rangle .
\end{gathered}
$$


Here, Eq. (C6) describes the contribution to the field due to stimulated emission of atom $a$. This results in amplification for the case when the atom is in an inverted state $\left\langle\hat{\sigma}_{z}^{(a)}\right\rangle>0$ and in absorption otherwise; Eq. (C7) describes the contribution to the field due to spontaneous emission of atom $a$. Evaluation of the spontaneous emission requires the knowledge of the one-atom two-time correlation function for atomic coherences. With the help of Eq. (C3), we can express it as

$$
\begin{gathered}
\left\langle\hat{\sigma}_{+}^{(a)}\left(\tau_{1}\right) \hat{\sigma}_{-}^{(a)}\left(\tau_{2}\right)\right\rangle=\left\langle\hat{\sigma}_{+}^{(a)}\left(\tau_{1}\right) \hat{\sigma}_{-}^{(a)}\left(\tau_{2}\right)\right\rangle_{\text {free }}+\left\langle\hat{\sigma}_{+}^{(a)}\left(\tau_{1}\right) \hat{\sigma}_{-}^{(a)}\left(\tau_{2}\right)\right\rangle_{\text {stim }}, \\
\left\langle\hat{\sigma}_{+}^{(a)}\left(\tau_{1}\right) \hat{\sigma}_{-}^{(a)}\left(\tau_{2}\right)\right\rangle_{\text {free }}=\rho_{e e}^{(a)}(0) e^{i \Omega\left(\tau_{1}-\tau_{2}\right)} e^{-\frac{\Gamma_{\mathrm{sp}}}{2}\left(\tau_{1}+\tau_{2}\right)}, \\
\left\langle\hat{\sigma}_{+}^{(a)}\left(\tau_{1}\right) \hat{\sigma}_{-}^{(a)}\left(\tau_{2}\right)\right\rangle_{\mathrm{stim}}=\frac{3 \Delta o \lambda^{2}}{2 \pi} \Gamma_{\mathrm{sp}} \int_{0}^{\tau_{1}} d \tau_{1}^{\prime} \int_{0}^{\tau_{2}} d \tau_{2}^{\prime} e^{i \Omega\left(\tau_{1}-\tau_{1}^{\prime}-\left[\tau_{2}-\tau_{2}^{\prime}\right]\right)} e^{-\frac{\Gamma_{\mathrm{sp}}}{2}\left(\tau_{1}-\tau_{1}^{\prime}+\tau_{2}-\tau_{2}^{\prime}\right)}\left\langle\hat{\sigma}_{z}^{(a)}\left(\tau_{1}^{\prime}\right) \hat{\sigma}_{z}^{(a)}\left(\tau_{2}^{\prime}\right)\right\rangle G^{(a)}\left(\tau_{1}^{\prime}, \tau_{2}^{\prime}\right) .
\end{gathered}
$$

Here, Eq. (C9) gives the value of $\left\langle\hat{\sigma}_{+}^{(a)}\left(\tau_{1}\right) \hat{\sigma}_{-}^{(a)}\left(\tau_{2}\right)\right\rangle$ in the case of a free atom unaffected by any external field, while Eq. (C10) provides a correction due to the exposure to a field with a given correlation function. Exact evaluation of Eq. (C10) would require the knowledge of the single-atom two-time quantity $\left\langle\hat{\sigma}_{z}^{(a)}\left(\tau_{1}^{\prime}\right) \hat{\sigma}_{z}^{(a)}\left(\tau_{2}^{\prime}\right)\right\rangle$, which would in turn need a separate set of equations. However, the contribution of this term only becomes important, once the field reaches significant value, at which point the stimulated contribution Eq. (C6) already dominate over the spontaneous term of Eq. (C7). Hence, there is no need to calculate the correction Eq. (C10) to an anyway negligible term Eq. (C7).

The same conclusion can be drawn from the following quantitative argument. Let us make an order-of-magnitude estimate for Eqs. (C7), (C6), (C9), and (C10):

$$
\begin{aligned}
\delta G_{\mathrm{stim}} & \sim \Delta o \Gamma_{\mathrm{sp}} \tau G \rho_{\mathrm{inv}}, \\
\delta G_{\mathrm{sp}} & \sim \frac{\Delta o}{\lambda^{2}} \Gamma_{\mathrm{sp}} \rho_{e e}, \\
\left\langle\hat{\sigma}_{+} \hat{\sigma}_{-}\right\rangle_{\mathrm{stim}} & \sim \Delta o \lambda^{2} \Gamma_{\mathrm{sp}} \tau^{2} G \rho_{\mathrm{inv}}^{2}, \\
\left\langle\hat{\sigma}_{+} \hat{\sigma}_{-}\right\rangle_{\mathrm{sp}} & \sim \rho_{e e} .
\end{aligned}
$$

Here, $G, \tau, \rho_{\text {inv }}$ are typical values of the field correlation function, evolution time and population inversion, respectively. From Eqs. (C11)-(C14) we obtain the following relation:

$$
\frac{\left\langle\hat{\sigma}_{+} \hat{\sigma}_{-}\right\rangle_{\mathrm{stim}}}{\left\langle\hat{\sigma}_{+} \hat{\sigma}_{-}\right\rangle_{\mathrm{sp}}} \sim \frac{\delta G_{\mathrm{stim}}}{\delta G_{\mathrm{sp}}} \Delta o \rho_{\mathrm{inv}} \Gamma_{\mathrm{sp}} \tau
$$

Accurate calculation of Eq. (C10) is important only while Eq. (C6) is smaller than Eq. (C7), hence we can set

$$
\frac{\delta G_{\text {stim }}}{\delta G_{\text {sp }}} \lesssim 1
$$

in Eq. (C15). The typical time to build up the field that would dominate over the spontaneous emission can be estimated as the superradiance time $\tau_{\mathrm{SR}} \sim \frac{1}{\Gamma_{\mathrm{sp}} \rho_{\text {inv }} n L \Delta o}$, here $n L$ is the number of atoms in the medium. Hence, while we assume that Eq. (C16) holds, we can take

$$
\tau \lesssim \frac{1}{\Gamma_{\text {sp }} \rho_{\text {inv }} n L \Delta o} .
$$

In the frame of Eqs. (C16) and (C17) we straightforwardly find with Eq. (C15)

$$
\frac{\left\langle\hat{\sigma}_{+} \hat{\sigma}_{-}\right\rangle_{\text {stim }}}{\left\langle\hat{\sigma}_{+} \hat{\sigma}_{-}\right\rangle_{\mathrm{sp}}} \lesssim \frac{1}{n L} \ll 1 .
$$

This shows that in the region, where Eq. (C7) is important, we can neglect the contribution of Eq. (C10) with respect to Eq. (C9). This conclusion was further supported by numerical calculation of the ratio between Eq. (C9) and the estimate of Eq. (C10). Another way of checking it is to calculate the intensity directly from atomic variables Eq. (20) and alternatively from the field correlation function $I^{(a)}(\tau)=G^{(a)}(\tau, \tau)$. In the first case, no approximation with regard to Eq. (C10) is made and its results agree with the approximate method. Hence, we will make the approximation of dropping off the contribution Eq. (C10) and arrive at Eq. (22).

\section{APPENDIX D: MODIFICATION OF EQUATIONS FOR ATOMIC AND FIELD OBSERVABLES DUE TO INCOHERENT PROCESSES}

Here, we consider how the expressions that were used to derive Eqs. (24)-(26) change in the presence of incoherent processes described by Lindblad superoperator Eq. (34).

The interaction with a Markovian reservoir can be described within the frame of Heisenberg-Langevin equations by the addition of a regular and a stochastic operator; see Ref. [39], Ch. 9. Hence, equations of the form of Eqs. (11) and (12) are transformed into

$$
\begin{gathered}
\frac{d \hat{O}_{\mu}^{(a)}(\tau)}{d \tau}=\left(\frac{d \hat{O}_{\mu}^{(a)}(\tau)}{d \tau}\right)_{\text {field }}+\hat{V}_{\mu}^{(a)}(\tau)+\hat{F}_{\mu}^{(a)}(\tau), \\
\left\langle\hat{F}_{\mu}^{(a)}(\tau)\right\rangle=0, \\
\left\langle\hat{F}_{\mu}^{(a)}\left(\tau_{1}\right) \hat{F}_{\nu}^{(b)}\left(\tau_{2}\right)\right\rangle=D_{\mu \nu}\left(\tau_{1}\right) \delta_{a b} \delta\left(\tau_{1}-\tau_{2}\right) .
\end{gathered}
$$

Here, $\hat{O}_{\mu}$ stands for some set of operators, e.g., $\sigma_{+}, \sigma_{-}, \sigma_{z}$; the first term comprises the evolution due to spontaneous decay and the interaction between atoms via their emitted field; the second and the third terms are regular and noise (Langevin) contributions due to the incoherent processes; the Kronecker symbol $\delta_{a b}$ in Eq. (D2) reflects our assumption that noise terms are uncorrelated if they correspond to different atoms (uncorrelated reservoirs).

If one knows the solution of master equation for one-atom density matrix, the value of any one-atom observable can be obtained directly from it; see Ref. [39], Ch. 9. Also, from the 
definition of quantum-mechanical mean we have

$$
\frac{d\left\langle\hat{O}_{\mu}^{(a)}(\tau)\right\rangle}{d \tau}=\left[\frac{d\left\langle\hat{O}_{\mu}^{(a)}(\tau)\right\rangle}{d \tau}\right]_{\text {field }}+\operatorname{Tr}\left[\hat{O}_{\mu}^{(a)}(\tau)\left(\frac{\partial \hat{\rho}^{(a)}}{\partial \tau}\right)_{\text {incoh }}\right] .
$$

The last term can be presented as

$$
\begin{aligned}
\operatorname{Tr}\left[\hat{O}_{\mu}^{(a)}(\tau)\left(\frac{\partial \hat{\rho}^{(a)}}{\partial \tau}\right)_{\text {incoh }}\right] & =\operatorname{Tr}\left[\hat{O}_{\mu}^{(a)}(\tau) L^{(a)}\left\{\hat{\rho}^{(a)}\right\}\right] \\
& =\operatorname{Tr}\left[\hat{V}_{\mu}^{(a)}(\tau) \hat{\rho}^{(a)}\right] .
\end{aligned}
$$

We can now obtain $\hat{V}_{\mu}^{(a)}(\tau)$ from the last equation using the actual form of the Lindblad superoperator Eq. (34) and taking into account that we can perform cyclic permutation of operators under the trace.

\section{Equations for $\rho_{e e}, \rho_{g g}$}

In this part, we outline how the equation for the population inversion would modify. In contrast to the case of a pure two level system considered in Sec. II, the identity $\rho_{e e}^{(a)}+$ $\rho_{g g}^{(a)}=1$ is not satisfied in general case. Hence, we have to obtain separate equations for $\rho_{e e}^{(a)}(\tau)=\left\langle\hat{\sigma}_{+}^{(a)}(\tau) \hat{\sigma}_{-}^{(a)}(\tau)\right\rangle$ and $\rho_{g g}^{(a)}(\tau)=\left\langle\hat{\sigma}_{-}^{(a)}(\tau) \hat{\sigma}_{+}^{(a)}(\tau)\right\rangle$. Using these operators in Eq. (D4) we obtain

$$
\begin{aligned}
& \left\langle\hat{V}_{e e}^{(a)}(\tau)\right\rangle=r_{e}\left(z_{a}, \tau\right)-\left(\gamma_{e}\left(z_{a}, \tau\right)+\gamma_{n}\right) \rho_{e e}^{(a)}(\tau), \\
& \left\langle\hat{V}_{g g}^{(a)}(\tau)\right\rangle=r_{g}\left(z_{a}, \tau\right)+\gamma_{n} \rho_{e e}^{(a)}(\tau)-\gamma_{g}\left(z_{a}, \tau\right) \rho_{g g}^{(a)}(\tau) .
\end{aligned}
$$

Here, $r_{e, g}\left(z_{a}, \tau\right)=\tilde{r}_{e, g}\left(z_{a}, \tau\right) \rho_{x x}^{(a)}(\tau)$ is the pumping rate of level $|e\rangle$ or $|g\rangle$, which is proportional to the occupation of the state $|x\rangle$, from which the pumping takes place. Taking into account that contributions due to spontaneous decay and interaction with emitted field can be expressed as

$$
\begin{aligned}
& \left(\frac{d \rho_{e e}^{(a)}(\tau)}{d \tau}\right)_{\text {field }}=\left(\frac{d\left\langle\hat{\sigma}_{z}^{(a)}(\tau)\right\rangle}{d \tau}\right)_{\text {field }}, \\
& \left(\frac{d \rho_{g g}^{(a)}(\tau)}{d \tau}\right)_{\text {field }}=-\left(\frac{d\left\langle\hat{\sigma}_{z}^{(a)}(\tau)\right\rangle}{d \tau}\right)_{\text {field }},
\end{aligned}
$$

we obtain as new equations for the continuous variables:

$$
\begin{aligned}
\frac{\partial \rho_{e e}(z, \tau)}{\partial \tau}= & r_{e}(z, \tau)-\left(\Gamma_{\mathrm{sp}}+\gamma_{e}(z, \tau)+\gamma_{n}\right) \rho_{e e}(z, \tau) \\
& -\frac{3 \Delta o}{8 \pi} \Gamma_{\mathrm{sp}} n \int_{0}^{z} d z^{\prime} S\left(z, z^{\prime}, \tau\right), \\
\Gamma_{e e}(z, \tau)= & \Gamma_{\mathrm{sp}}+\gamma_{e}(z, \tau)+\gamma_{n}, \\
\frac{\partial \rho_{g g}(z, \tau)}{\partial \tau}= & r_{g}(z, \tau)+\left(\Gamma_{\mathrm{sp}}+\gamma_{n}\right) \rho_{e e}(z, \tau)-\gamma_{g}(z, \tau) \rho_{g g}(z, \tau) \\
& +\frac{3 \Delta o}{8 \pi} \Gamma_{\mathrm{sp}} n \int_{0}^{z} d z^{\prime} S\left(z, z^{\prime}, \tau\right) .
\end{aligned}
$$

\section{Equations for $S\left(z_{1}, z_{2}, \tau\right)$} find

From expression Eq. (D4) applied to operators $\hat{\sigma}_{ \pm}^{(a)}$, we $\hat{V}_{ \pm}^{(a)}(\tau)=-\frac{1}{2}\left[\gamma_{n}+q\left(z_{a}, \tau\right)+\gamma_{e}\left(z_{a}, \tau\right)+\gamma_{g}\left(z_{a}, \tau\right)\right] \hat{\sigma}_{ \pm}^{(a)}(\tau)$.

Hence, in equations involving $\hat{\sigma}_{ \pm}^{(a)}$ operators, such as Eq. (11), the account of Eq. (D9) is equivalent to modifying the decay term by changing the decay rate to

$$
\Gamma\left(z_{a}, \tau\right)=\Gamma_{\mathrm{sp}}+\gamma_{n}+q\left(z_{a}, \tau\right)+\gamma_{e}\left(z_{a}, \tau\right)+\gamma_{g}\left(z_{a}, \tau\right) .
$$

Equation (11) and its hermitian conjugate were used to obtain the equation for the time-propagation of the correlation function of atomic coherences Eq. (16). Taking into account that the derivation was based on quantum Einstein relation Eq. (B2), one can see that the inclusion of additional operators Eq. (D9) results in the following substitution of the term $-\Gamma_{\mathrm{sp}} S\left(z_{1}, z_{2}, \tau\right)$ in Eq. (25) by

$$
-\Gamma_{\mathrm{sp}} S\left(z_{1}, z_{2}, \tau\right) \rightarrow-\frac{1}{2}\left[\Gamma\left(z_{1}, \tau\right)+\Gamma\left(z_{2}, \tau\right)\right] S\left(z_{1}, z_{2}, \tau\right) .
$$

Note that the term $D_{12}$ in Eq. (B2) gives no contribution due to our assumption that the reservoirs are independent for each atom.

\section{Equations for the field correlation function}

Finally, we discuss, which modifications need to be introduced to the derivation done in Appendix C. In Eq. (C3), we have to take into account the additional terms from Eq. (D9); this results in

$$
\begin{aligned}
\hat{\sigma}_{-}^{(a)}(\tau)= & \int_{0}^{\tau} d \tau^{\prime} e^{-i \Omega\left(\tau-\tau^{\prime}\right)} e^{-\frac{1}{2} \int_{\tau^{\prime}}^{\tau} d \tau^{\prime \prime} \Gamma\left(z_{a}, \tau^{\prime \prime}\right)} \\
& \times\left[\frac{2 i e p}{\hbar m c} \hat{\sigma}_{z}^{(a)}\left(\tau^{\prime}\right) \hat{A}_{+}^{(a)}\left(\tau^{\prime}\right)+\hat{F}_{-}^{(a)}\left(\tau^{\prime}\right)\right] \\
& +e^{-i \Omega \tau} e^{-\frac{1}{2} \int_{0}^{\tau} d \tau^{\prime \prime} \Gamma\left(z_{a}, \tau^{\prime \prime}\right)} \hat{\sigma}_{-}^{(a)}(0) .
\end{aligned}
$$

Retaining the damping term $e^{-\frac{1}{2} \int_{\tau^{\prime}}^{\tau} d \tau^{\prime \prime} \Gamma\left(z_{a}, \tau^{\prime \prime}\right)}$ throughout further derivations results in the factor $\mathcal{D}$ appearing in Eq. (39).

Additional modifications affect the quantity $\left\langle\hat{\sigma}_{+}^{(a)}\left(\tau_{1}\right) \hat{\sigma}_{-}^{(a)}\left(\tau_{2}\right)\right\rangle$, which is needed to calculate Eq. (C7). Following the same reasoning as in Appendix $\mathrm{C}$ we consider only contributions from $\left\langle\hat{\sigma}_{+}^{(a)}\left(\tau_{1}\right) \hat{\sigma}_{-}^{(a)}\left(\tau_{2}\right)\right\rangle_{\text {free }}$, i.e., evolution only due to the spontaneous decay and incoherent processes of individual atoms. To this end, we use Eq. (D12) omitting the field and write

$$
\begin{aligned}
\left\langle\hat{\sigma}_{+}^{(a)}\left(\tau_{1}\right) \hat{\sigma}_{-}^{(a)}\left(\tau_{2}\right)\right\rangle= & \int_{0}^{\tau_{1}} d \tau_{1}^{\prime} \int_{0}^{\tau_{2}} d \tau_{2}^{\prime} e^{i \Omega\left(\tau_{1}-\tau_{1}^{\prime}-\left[\tau_{2}-\tau_{2}^{\prime}\right]\right)} e^{-\frac{1}{2}\left[\int_{\tau_{1}^{\prime}}^{\tau_{1}} d \tau_{1}^{\prime \prime} \Gamma\left(z_{a}, \tau_{1}^{\prime \prime}\right)+\int_{\tau_{2}}^{\tau_{2}} d \tau_{2}^{\prime \prime} \Gamma\left(z_{a}, \tau_{2}^{\prime \prime}\right)\right]}\left\langle\hat{F}_{+}^{(a)}\left(\tau_{1}^{\prime}\right) \hat{F}_{-}^{(a)}\left(\tau_{2}^{\prime}\right)\right\rangle \\
& +e^{-\frac{1}{2}\left[\int_{0}^{\tau_{1}} d \tau_{1}^{\prime \prime} \Gamma\left(z_{a}, \tau_{1}^{\prime \prime}\right)+\int_{0}^{\tau_{2}} d \tau_{2}^{\prime \prime} \Gamma\left(z_{a}, \tau_{2}^{\prime \prime}\right)\right]} e^{i \Omega\left(\tau_{1}-\tau_{2}\right)} \rho_{e e}^{(a)}(0) .
\end{aligned}
$$


To evaluate Eq. (D13), we need to know the value of the noise correlation $D_{+-}(\tau)$; see Eq. (D2). It can be obtained from the combination of the master equation and the generalized Einstein relation Eq. (B2), see Ref. [39] Ch. 9.4:

$$
\begin{aligned}
D_{+-}(\tau) & =\left\langle\hat{V}_{e e}^{(a)}(\tau)\right\rangle-\left\langle\hat{V}_{+}^{(a)}(\tau) \hat{\sigma}_{-}^{(a)}(\tau)\right\rangle-\left\langle\hat{\sigma}_{+}^{(a)}(\tau) \hat{V}_{-}^{(a)}(\tau)\right\rangle \\
& =r_{e}\left(z_{a}, \tau\right)+\left[\Gamma\left(z_{a}, \tau\right)-\Gamma_{e e}\left(z_{a}, \tau\right)\right] \rho_{e e}^{(a)}(\tau) .
\end{aligned}
$$

With the help of Eq. (D2) and using Eq. (D14), Eq. (D13) transforms to

$$
\begin{aligned}
& \left\langle\hat{\sigma}_{+}^{(a)}\left(\tau_{1}\right) \hat{\sigma}_{-}^{(a)}\left(\tau_{2}\right)\right\rangle \\
& \quad=e^{i \Omega\left(\tau_{1}-\tau_{2}\right)} \int_{0}^{\min \tau_{1}, \tau_{2}} d \tau^{\prime} e^{-\frac{1}{2}\left[\int_{\tau^{\prime}}^{\tau_{1}} d \tau_{1}^{\prime \prime} \Gamma\left(z_{a}, \tau_{1}^{\prime \prime}\right)+\int_{\tau^{\prime}}^{\tau_{2}} d \tau_{2}^{\prime \prime} \Gamma\left(z_{a}, \tau_{2}^{\prime \prime}\right)\right]}
\end{aligned}
$$$$
\rho_{g e}\left(z_{1}, \tau\right) \frac{\partial \rho_{e g}\left(z_{2}, \tau\right)}{\partial \tau}+\rho_{e g}\left(z_{2}, \tau\right) \frac{\partial \rho_{g e}\left(z_{1}, \tau\right)}{\partial \tau}
$$$$
=\rho_{g e}\left(z_{1}, \tau\right)\left[-\frac{\Gamma\left(z_{2}, \tau\right)}{2} \rho_{e g}\left(z_{2}, \tau\right)+\frac{3 \Delta o}{16 \pi} \Gamma_{\mathrm{sp}} \rho_{\mathrm{inv}}\left(z_{2}, \tau\right) n \int_{0}^{z_{2}} d z_{2}^{\prime} \mathcal{A}\left(z_{2}, z_{2}^{\prime}\right) \rho_{e g}\left(z_{2}^{\prime}, \tau\right)\right]
$$$$
\times \rho_{e g}\left(z_{2}, \tau\right)\left[-\frac{\Gamma\left(z_{1}, \tau\right)}{2} \rho_{g e}\left(z_{1}, \tau\right)+\frac{3 \Delta o}{16 \pi} \Gamma_{\mathrm{sp}} \rho_{\mathrm{inv}}\left(z_{1}, \tau\right) n \int_{0}^{z_{1}} d z_{1}^{\prime} \mathcal{A}\left(z_{1}, z_{1}^{\prime}\right) \rho_{g e}\left(z_{1}^{\prime}, \tau\right)\right] .
$$

$$
\begin{aligned}
& \times\left[r_{e}\left(z_{a}, \tau^{\prime}\right)+\left[\Gamma\left(z_{a}, \tau^{\prime}\right)-\Gamma_{e e}\left(z_{a}, \tau^{\prime}\right)\right] \rho_{e e}^{(a)}\left(\tau^{\prime}\right)\right] \\
& +e^{i \Omega\left(\tau_{1}-\tau_{2}\right)} e^{-\frac{1}{2}\left[\int_{0}^{\tau_{1}} d \tau_{1}^{\prime \prime} \Gamma\left(z_{a}, \tau_{1}^{\prime \prime}\right)+\int_{0}^{\tau_{2}} d \tau_{2}^{\prime \prime} \Gamma\left(z_{a}, \tau_{2}^{\prime \prime}\right)\right]} \rho_{e e}^{(a)}(0) .
\end{aligned}
$$

This constitutes the spontaneous emission part of Eq. (39).

\section{APPENDIX E: DERIVATION OF MAXWELL-BLOCH EQUATIONS FROM THE CORRELATION FUNCTION EQUATIONS}

Here, we consider how the correlation function Eqs. (36)(39) simplify if we assume the factorization of Eqs. (46) and (47) and omit the spontaneous emission terms. Under these assumptions, we can substitute Eq. (46) into Eq. (38) and obtain the following expression:
This itself can obviously be expressed in factorized form as well. The integral in Eq. (E1) can be represented as the solution of a differential equation and in this way, we can introduce an electric field envelope that satisfies

$$
\begin{aligned}
\frac{\partial \mathcal{E}_{+}(z, \tau)}{\partial z} & =-\frac{\kappa(z)}{2} \mathcal{E}_{+}(z, \tau)+\frac{i \Omega}{2 \epsilon_{0} c} \mu n_{v} \rho_{e g}(z, \tau) \xi, \\
\xi & =\frac{\Delta o \pi R^{2}}{2 \lambda^{2}} .
\end{aligned}
$$

Using this, we obtain from Eq. (E1)

$$
\frac{\partial \rho_{g e}(z, \tau)}{\partial \tau}=-\frac{\Gamma(z, \tau)}{2} \rho_{g e}(z, \tau)+\frac{i \mu}{\hbar} \rho_{\text {inv }}(z, \tau) \mathcal{E}_{-}(z, \tau) .
$$

Here, we used the definition of the spontaneous decay rate Eq. (A9), and the dipole moment $\mu$ that is related to the momentum matrix element given in Eq. (4) as $e p=i m \Omega \mu$. Furthermore, $n_{v}$ is the three-dimensional particle density, namely $n=n_{v} \pi R^{2}$, while $\mathcal{E}_{+}$and $\mathcal{E}_{-}$are the positive and negative frequency parts of the field; they are complex conjugate to one another. The way how to split the factor $3 \Delta o /(16 \pi) \Gamma_{\mathrm{sp}}$ into prefactors in Eqs. (E2) and (E3) is arbitrary at this stage. The chosen factors comply with Eqs. (5) and (10). Since these expressions are our basic expressions to obtain field correlation function Eq. (39), the field propagation Eq. (E2) should agree with Eqs. (39) and (47). Notably, the semiclassical Maxwell-Bloch equations could also be obtained directly from Eqs. (5), (6), (10), and (D9) by replacing all operators by $c$-numbers and omitting the operator noise terms.

The field Eq. (E2) differs from typically used MaxwellBloch equations [18] by a factor $\xi$. This is result of the 1D approximation Eq. (8), though within this approximation, it can be taken as $\xi \sim 1$. With this choice we obtain Eqs. (48)(51).

\section{APPENDIX F: NOISE-TERM CORRELATION FACTOR FOR MAXWELL-BLOCH EQUATIONS}

To compare the present approach to Maxwell-Bloch equations with noise terms we derive the noise term correlation factor $F$ that is consistent with the approximations and conventions used throughout this paper. We restrict ourselves to a simplified scheme, assuming instantaneous pumping and the absence of incoherent processes except for decoherence with a fixed decoherence rate $q$. If no external field is present, then from Eq. (E3) supplemented with the noise term $s_{+}(z, \tau)$ we obtain

$$
\rho_{g e}(z, \tau)=\int_{0}^{\tau} d \tau^{\prime} e^{-\frac{\Gamma}{2}\left(\tau-\tau^{\prime}\right)} s_{+}\left(z, \tau^{\prime}\right)
$$

where $\Gamma=\Gamma_{\mathrm{sp}}+q$. Substituting this expression into Eq. (E2) and considering the case without absorption, we find

$$
\mathcal{E}_{+}(z, \tau)=\frac{i \Omega \mu n_{v}}{2 \epsilon_{0} c} \int_{0}^{z} d z^{\prime} \int_{0}^{\tau} d \tau^{\prime} e^{-\frac{\Gamma}{2}\left(\tau-\tau^{\prime}\right)} s_{-}\left(z^{\prime}, \tau^{\prime}\right),
$$

with $\xi \sim 1$.

The number of spontaneously emitted photons can be obtained as

$$
\frac{d N_{\mathrm{ph}, \mathrm{sp}}(\tau)}{d \tau}=\frac{2 \epsilon_{0} c}{\hbar \omega}\left\langle\mathcal{E}_{-}(\tau) \mathcal{E}_{+}(\tau)\right\rangle \pi R^{2},
$$

where $\langle.$.$\rangle indicates the average over realizations of the$ stochastic variables $s_{ \pm}(z, \tau)$. Substituting Eq. (F2) into Eq. (F3), taking into 
account Eq. (52), performing integrals with the $\delta$ functions and using Eq. (A9), we arrive at

$$
\frac{d N_{\mathrm{ph}, \mathrm{sp}}(\tau)}{d \tau}=\frac{3}{8 \pi} \Gamma_{\mathrm{sp}} n z n_{v} F \lambda^{2} \int_{0}^{\tau} d \tau^{\prime} e^{-\Gamma\left(\tau-\tau^{\prime}\right)} \rho_{e e}\left(\tau^{\prime}\right) .
$$

We can now compare the obtained expression with the exact result Eq. (43). If we assume that the decoherence time is much shorter than the spontaneous emission time, we can take $\rho_{e e}(\tau)$ out of the integral and obtain

$$
F=\frac{2 \Gamma}{n} \xi
$$

Here again, we can take $\xi \sim 1$. However, if $\Gamma=\Gamma_{\mathrm{sp}}$, then within the choice Eq. (F5) for the temporal dependence of spontaneously emitted quanta we find

$$
\frac{d N_{\mathrm{ph}, \mathrm{sp}}(\tau)}{d \tau}=\frac{3}{8 \pi} \Gamma_{\mathrm{sp}} n z \Delta o \rho_{e e}(0) \Gamma_{\mathrm{sp}} \tau e^{-\Gamma_{\mathrm{sp}} \tau},
$$

under the same approximation $\xi \sim 1$. Clearly, Eq. (F6) differs from the correct result by a factor $\Gamma_{\mathrm{sp}} \tau$.
[1] M. A. Duguay and P. M. Rentzepis, Appl. Phys. Lett. 10, 350 (1967).

[2] J. J. Rocca, Rev. Sci. Instrum. 70, 3799 (1999).

[3] W. T. Silfvast, J. J. Macklin, and O. R. Wood, Opt. Lett. 8, 551 (1983).

[4] D. L. Matthews, P. L. Hagelstein, M. D. Rosen, M. J. Eckart, N. M. Ceglio, A. U. Hazi, H. Medecki, B. J. MacGowan, J. E. Trebes, B. L. Whitten, E. M. Campbell, C. W. Hatcher, A. M. Hawryluk, R. L. Kauffman, L. D. Pleasance, G. Rambach, J. H. Scofield, G. Stone, and T. A. Weaver, Phys. Rev. Lett. 54, 110 (1985).

[5] Y. Nagata, K. Midorikawa, S. Kubodera, M. Obara, H. Tashiro, and K. Toyoda, Phys. Rev. Lett. 71, 3774 (1993).

[6] H. Daido, Rep. Prog. Phys. 65, 1513 (2002).

[7] S. Suckewer and P. Jaeglé, Laser Phys. Lett. 6, 411 (2009).

[8] N. Rohringer, D. Ryan, R. A. London, M. Purvis, F. Albert, J. Dunn, J. D. Bozek, C. Bostedt, A. Graf, R. Hill, S. P. Hau-Riege, and J. J. Rocca, Nature 481, 488 (2012).

[9] H. Yoneda, Y. Inubushi, K. Nagamine, Y. Michine, H. Ohashi, H. Yumoto, K. Yamauchi, H. Mimura, H. Kitamura, T. Katayama, T. Ishikawa, and M. Yabashi, Nature 524, 446 (2015).

[10] T. Kroll, C. Weninger, R. Alonso-Mori, D. Sokaras, D. Zhu, L. Mercadier, V. P. Majety, A. Marinelli, A. Lutman, M. W. Guetg, F.-J. Decker, S. Boutet, A. Aquila, J. Koglin, J. Koralek, D. P. DePonte, J. Kern, F. D. Fuller, E. Pastor, T. Fransson, Y. Zhang, J. Yano, V. K. Yachandra, N. Rohringer, and U. Bergmann, Phys. Rev. Lett. 120, 133203 (2018).

[11] C. Weninger, M. Purvis, D. Ryan, R. A. London, J. D. Bozek, C. Bostedt, A. Graf, G. Brown, J. J. Rocca, and N. Rohringer, Phys. Rev. Lett. 111, 233902 (2013).

[12] C. Weninger and N. Rohringer, Phys. Rev. A 90, 063828 (2014).

[13] N. Skribanowitz, I. P. Herman, J. C. MacGillivray, and M. S. Feld, Phys. Rev. Lett. 30, 309 (1973).

[14] H. M. Gibbs, Q. H. F. Vrehen, and H. M. J. Hikspoors, Phys. Rev. Lett. 39, 547 (1977).

[15] Q. H. F. Vrehen and M. F. H. Schuurmans, Phys. Rev. Lett. 42, 224 (1979).

[16] M. S. Malcuit, J. J. Maki, D. J. Simkin, and R. W. Boyd, Phys. Rev. Lett. 59, 1189 (1987).

[17] R. H. Dicke, Phys. Rev. 93, 99 (1954).

[18] M. Gross and S. Haroche, Phys. Rep. 93, 301 (1982).

[19] M. G. Benedikt, A. M. Ermolaev, V. A. Malyshev, I. V. Sokolov, and E. D. Trifonov, Super-radiance: Multiatomic Coherent
Emission (Institute of Physics Publishing, Philadelphia, PA, 1996).

[20] V. V. Kocharovsky, V. V. Zheleznyakov, E. R. Kocharovskaya, and V. V. Kocharovsky, Phys. Usp. 60, 345 (2017).

[21] V. Kimberg and N. Rohringer, Phys. Rev. Lett. 110, 043901 (2013).

[22] C. Lyu, S. M. Cavaletto, C. H. Keitel, and Z. Harman, arXiv: 1801.02503.

[23] O. Larroche, D. Ros, A. Klisnick, A. Sureau, C. Möller, and H. Guennou, Phys. Rev. A 62, 043815 (2000).

[24] A. Depresseux, E. Oliva, J. Gautier, F. Tissandier, J. Nejdl, M. Kozlova, G. Maynard, J. P. Goddet, A. Tafzi, A. Lifschitz, H. T. Kim, S. Jacquemot, V. Malka, K. Ta Phuoc, C. Thaury, P. Rousseau, G. Iaquaniello, T. Lefrou, A. Flacco, B. Vodungbo, G. Lambert, A. Rousse, P. Zeitoun, and S. Sebban, Nat. Photon. 9, 817 (2015).

[25] E. Oliva, M. Fajardo, L. Li, M. Pittman, T. T. T. Le, J. Gautier, G. Lambert, P. Velarde, D. Ros, S. Sebban, and P. Zeitoun, Nat. Photon. 6, 764 (2012).

[26] Y. Wang, S. Wang, E. Oliva, L. Li, M. Berrill, L. Yin, J. Nejdl, B. M. Luther, C. Proux, T. T. T. Le, J. Dunn, D. Ros, P. Zeitoun, and J. J. Rocca, Nat. Photon. 8, 381 (2014).

[27] A. Depresseux, E. Oliva, J. Gautier, F. Tissandier, G. Lambert, B. Vodungbo, J.-P. Goddet, A. Tafzi, J. Nejdl, M. Kozlova, G. Maynard, H. T. Kim, K. T. Phuoc, A. Rousse, P. Zeitoun, and S. Sebban, Phys. Rev. Lett. 115, 083901 (2015).

[28] N. E. Rehler and J. H. Eberly, Phys. Rev. A 3, 1735 (1971).

[29] R. Bonifacio and L. A. Lugiato, Phys. Rev. A 11, 1507 (1975).

[30] R. Bonifacio and L. A. Lugiato, Phys. Rev. A 12, 587 (1975).

[31] J. C. MacGillivray and M. S. Feld, Phys. Rev. A 14, 1169 (1976).

[32] D. Polder, M. F. H. Schuurmans, and Q. H. F. Vrehen, Phys. Rev. A 19, 1192 (1979).

[33] F. Haake, H. King, G. Schröder, J. Haus, and R. Glauber, Phys. Rev. A 20, 2047 (1979).

[34] J. J. Maki, M. S. Malcuit, M. G. Raymer, R. W. Boyd, and P. D. Drummond, Phys. Rev. A 40, 5135 (1989).

[35] P. D. Drummond and M. G. Raymer, Phys. Rev. A 44, 2072 (1991).

[36] F. A. Hopf, P. Meystre, M. O. Scully, and J. F. Seely, Phys. Rev. Lett. 35, 511 (1975).

[37] F. A. Hopf and P. Meystre, Phys. Rev. A 12, 2534 (1975).

[38] W. H. Louisell, M. O. Scully, and W. B. McKnight, Phys. Rev. A 11, 989 (1975). 
[39] M. O. Scully and M. S. Zubairy, Quantum Optics (Cambridge University Press, Cambridge, 1997).

[40] M. Altarelli, Resonant X-ray scattering: A theoretical introduction, in Magnetism: A Synchrotron Radiation Approach, edited by E. Beaurepaire, H. Bulou, F. Scheurer, and J.-P. Kappler (Springer, Berlin/Heidelberg, 2006), pp. 218-219.

[41] G. S. Agarwal, Quantum statistical theories of spontaneous emission and their relation to other approaches, in Quantum Optics, edited by G. Höhler (Springer, Berlin/Heidelberg, 1974), pp. $1-128$.

[42] R. J. Glauber, Phys. Rev. 130, 2529 (1963).

[43] M. Bonitz, The method of reduced density operators, in Quantum Kinetic Theory (Springer International Publishing, Cham, 2016), pp. 29-69.

[44] D. Meiser and M. J. Holland, Phys. Rev. A 81, 033847 (2010).

[45] D. Meiser and M. J. Holland, Phys. Rev. A 81, 063827 (2010).

[46] E. Ressayre and A. Tallet, Phys. Rev. A 18, 2196 (1978).

[47] Z. Jurek, S.-K. Son, B. Ziaja, and R. Santra, J. Appl. Crystallogr. 49, 1048 (2016).

[48] E. J. McGuire, Phys. Rev. Lett. 35, 844 (1975).
[49] H. C. Kapteyn, R. W. Lee, and R. W. Falcone, Phys. Rev. Lett. 57, 2939 (1986).

[50] H. C. Kapteyn and R. W. Falcone, Phys. Rev. A 37, 2033 (1988).

[51] P. Meystre and M. Sargent, Elements of Quantum Optics (Springer-Verlag, Berlin/Heidelberg, 2007).

[52] H.-J. Briegel and B.-G. Englert, Phys. Rev. A 47, 3311 (1993).

[53] I. R. Al'miev, O. Larroche, D. Benredjem, J. Dubau, S. Kazamias, C. Möller, and A. Klisnick, Phys. Rev. Lett. 99, 123902 (2007).

[54] C. M. Kim, J. Lee, and K. A. Janulewicz, Phys. Rev. Lett. 104, 053901 (2010).

[55] S. Krušič, K. Bučar, A. Mihelič, and M. Žitnik, Phys. Rev. A 98, 013416 (2018).

[56] L. Mercadier, A. Benediktovitch, C. Weninger, M. A. Blessenohl, S. Bernitt, H. Bekker, S. Dobrodey, A. SánchezGonzález, B. Erk, C. Bomme, R. Boll, Z. Yin, V. P. Majety, R. Steinbrügge, M. A. Khalal, F. Penent, J. Palaudoux, P. Lablanquie, A. Rudenko, D. Rolles, J. R. C. López-Urrutia, and N. Rohringer, arXiv:1810.11097.

[57] L. Allen and J. H. Eberly, Optical Resonance and Two Level Atoms (Dover Publications, Mineola, NY, 1987). 